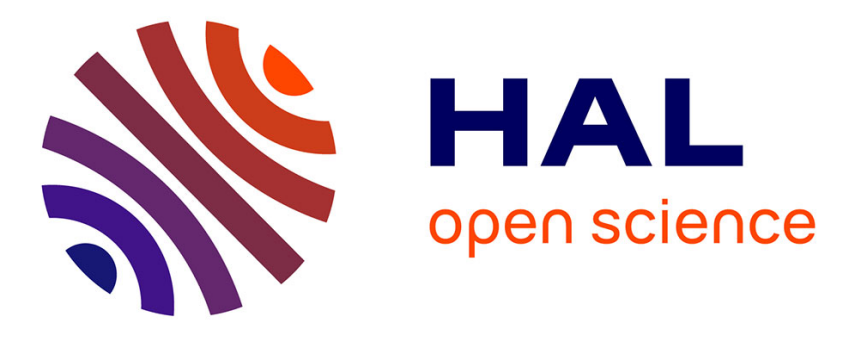

\title{
Shape optimization of fixed-bed reactors in process engineering
}

Alexis Courtais, Abderrazak Latifi, François Lesage, Yannick Privat

\section{To cite this version:}

Alexis Courtais, Abderrazak Latifi, François Lesage, Yannick Privat. Shape optimization of fixed-bed reactors in process engineering. SIAM Journal on Applied Mathematics, 2021, 81 (3), pp.1141-1165. 10.1137/20M1343841. hal-02819559v2

\section{HAL Id: hal-02819559 \\ https://hal.science/hal-02819559v2}

Submitted on 12 Mar 2021

HAL is a multi-disciplinary open access archive for the deposit and dissemination of scientific research documents, whether they are published or not. The documents may come from teaching and research institutions in France or abroad, or from public or private research centers.
L'archive ouverte pluridisciplinaire HAL, est destinée au dépôt et à la diffusion de documents scientifiques de niveau recherche, publiés ou non, émanant des établissements d'enseignement et de recherche français ou étrangers, des laboratoires publics ou privés. 


\title{
Shape optimization of fixed-bed reactors in process engineering
}

\author{
A. Courtais* \\ A. M. Latifi \\ F. Lesage $e^{\ddagger}$ \\ Y. Privat ${ }^{\S}$
}

\begin{abstract}
This paper deals with geometric shape optimization of a parallelepipedic fixed-bed reactor with single phase liquid flow where a chemical reaction takes place. The packing is a sort of static mixer made up of solid cylindrical obstacles uniformly distributed in the reactor. The reactive flow is described by means of momentum and mass transport equations in laminar flow regime. The objective is to determine the shape of the packing which maximizes the reaction conversion rate subjected to some specified manufacturing constraints. The optimization approach developed is based on the adjoint system method and the results show that the optimal shape obtained allows to significantly improve the reaction conversion rate. Furthermore, the optimal shape is printed by means of an additive manufacturing technique and several manufacturing constraints mainly related to the thickness of packing are considered.
\end{abstract}

Keywords: shape optimization, CFD, fixed-bed reactor, additive manufacturing.

AMS classification: 49M05, 65K10, 35Q30.

\section{Introduction}

Continuous improvement of the competitiveness of the chemical industry requires not only constant innovation, but also an inevitable evolution towards more intensive, efficient, compact and sustainable processes. The shape of the units (reactors, stirrers, packings, exchangers, pipes etc.) that make up an industrial process is one of the key parameters to improve competitiveness and represents a fundamental scientific and technological challenge.

Shape optimization was originally developed in fluid mechanics area [12], particularly in the design of aircraft wings in aerospace and aeronautics industries [32]. Indeed, shape optimization methods have been studied and developed since the 1960s and 1970s in this field [13, 25]. More recently, it has been used in process engineering to determine the optimal shape of a pipe/structure [24, 14] or a microchannel [36].

In chemical engineering however where the shape of unit operations is an important design parameter, approaches based on the so-called shape derivatives have not been extensively investigated. This important issue deserves therefore to be addressed and will probably result in a paradigm shift in optimal design and operation of processes. The chemical engineering literature naturally

\footnotetext{
*Laboratoire Réactions et Génie des Procédés, CNRS-ENSIC, Université de Lorraine, Nancy, France. (alexis.courtais@univ-lorraine.fr)

${ }_{\dagger}$ Laboratoire Réactions et Génie des Procédés, CNRS-ENSIC, Université de Lorraine, Nancy, France. (abderrazak.latifi@univ-lorraine.fr)

${ }^{\ddagger}$ Laboratoire Réactions et Génie des Procédés, CNRS-ENSIC, Université de Lorraine, Nancy, France. (francois.lesage@univ-lorraine.fr)

§IRMA, Université de Strasbourg, CNRS UMR 7501, 7 rue René Descartes, 67084 Strasbourg, France (yannick.privat@unistra.fr)
} 
contains multiple studies dedicated to problems of optimal design and shape optimization. Examples include $[6,22,28]$. However, the approach used does not generally allow to explore all possible forms and often comes down to a problem of optimizing a small number of parameters. The major drawback of these approaches is that they do not always allow substantial modifications of the shapes considered, and therefore do not lead to optimal performance.

Basically, there are three types of shape optimization: parametric, geometric and topologic [1]. In this paper, only geometric optimization is considered, meaning that all admissible designs have the same topology (roughly speaking, the same number of holes). The optimization approach developed in this study is based on the so-called shape derivative in the sense of Hadamard [23], using the adjoint system method. The case study is a $2 \mathrm{D}$ fixed-bed reactor with a laminar single phase liquid flow where a homogenous first order chemical reaction takes place. The packing is a kind of static mixer made up of solid cylindrical obstacles uniformly distributed in the reactor. The objective is to determine the shape of the packing which maximizes the reactor conversion rate. Moreover, when it comes to realistic shapes involved in industrial processes, the optimal shapes obtained must generally meet certain manufacturing constraints, which will depend a lot on the context of application envisaged. Sometimes this is done by post-processing the designed objects. In this article, 3D printing technology (i.e. additive manufacturing) is used to manufacture the optimal shape obtained taking into account the specified manufacturing constraints.

The paper is organized as follows: in Section 2, first principles model equations describing the liquid flow in the reactor are introduced. We first investigate an ideal version of the problem where the only manufacturing constraint considered is a volume constraint. We gather the needed material to compute optimal shapes, and introduce in Section 3 an algorithm based on the Hadamard shape derivative combined with an extension-regularization approach of the shape gradient to deal with mesh-displacement. This algorithm is then numerically tested on $2 \mathrm{D}$ examples in Section 4. We also propose a more intricate, but also more realistic version of the shape optimization problem, by introducing a constraint on the manufacturing of the optimal shape which will be achieved by means of an additive manufacturing technique. Additive manufacturing (or 3D printing) is opposed to subtractive manufacturing where material is removed to achieve the desired shape. In additive manufacturing, 3D parts are built by adding successive layers of material under computer control. Stratoconception, an additive conception method developed by the CIRTES ${ }^{1}$, is particularly well adapted to this use.

\section{Modeling and analysis of the shape optimization problem}

\section{$2.1 \quad$ Fixed-bed reactor modelling}

The fixed-bed reactor considered is a $2 \mathrm{D}$ packed bed with a single phase liquid flow where a homogenous first order chemical reaction takes place. The packing is made up of solid cylindrical obstacles (i.e. a kind of static mixer) uniformly distributed in the reactor. Figure 1 shows the schematic representation of the fixed-bed considered.

\footnotetext{
${ }^{1}$ namely, the European Center for Rapid Product Development,located in the heart of the Saint-Dié-des-Vosges industrial basin in France.
} 


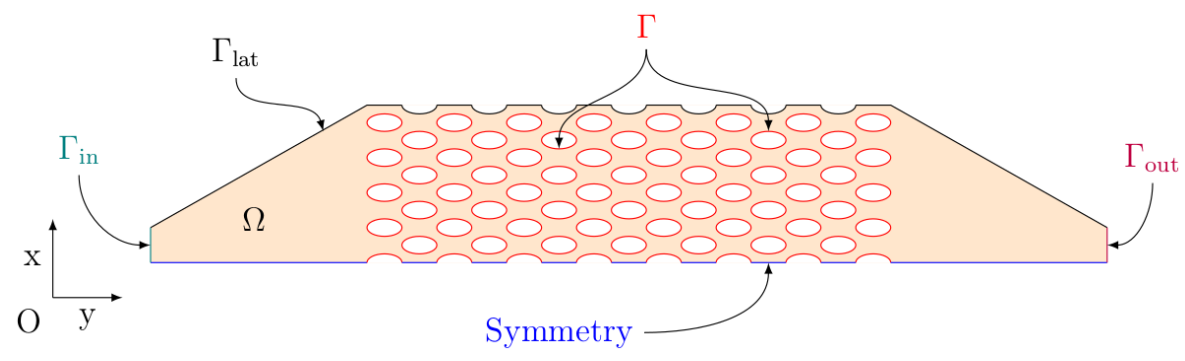

Figure 1: Schematic view of the fixed-bed reactor geometry (that we will use as initialization of the shape optimization algorithm). The "Symmetry" boundary refers to a symmetry axis present in the center of the reactor, symmetry boundary conditions are imposed on it.

The optimization method developed in this work is based on the process model equations describing the flow through the fixed-bed reactor. A two-dimensional model is therefore developed in laminar flow regime and involves mass and momentum balance equations. The reactor geometry is modeled by an open bounded domain $\Omega \subset \mathbb{R}^{2}$, whose boundaries are the union of the inlet $\Gamma_{i n}$, the outlet $\Gamma_{\text {out }}$, the lateral wall $\Gamma_{\text {lat }}$ and $\Gamma=\partial \Omega \backslash\left(\Gamma_{\text {in }} \cup \Gamma_{\text {out }} \cup \Gamma_{\text {lat }}\right)$, the free boundary. Roughly speaking, $\Gamma$ denotes the boundary of the pores inside the fixed-bed (the regular structure on Fig. 1).

The momentum transport is described by the following Navier-Stokes equations along with the associated boundary conditions:

$$
\begin{aligned}
-\nu \Delta \boldsymbol{U}+\boldsymbol{U} \cdot \nabla \boldsymbol{U}+\nabla p & =0 & & \text { in } \Omega \\
\nabla \cdot \boldsymbol{U} & =0 & & \text { in } \Omega \\
\boldsymbol{U} & =\boldsymbol{U}_{\boldsymbol{i n}} & & \text { on } \Gamma_{\text {in }} \\
\boldsymbol{U} & =0 & & \text { on } \Gamma_{\text {lat }} \cup \Gamma \\
\boldsymbol{\sigma}(\boldsymbol{U}, p) \boldsymbol{n} & =0 & & \text { on } \Gamma_{\text {out }}
\end{aligned}
$$

where $\nu>0$ denotes the kinematic viscosity of the fluid,

$$
\boldsymbol{\sigma}(\boldsymbol{U}, p)=2 \nu \varepsilon(\boldsymbol{U})-p \boldsymbol{I}, \text { with } \varepsilon(\boldsymbol{U})=\frac{1}{2}\left(\nabla \boldsymbol{U}+(\nabla \boldsymbol{U})^{\top}\right),
$$

is the viscous stress tensor divided by the fluid density, $p$ the fluid kinematic pressure (i.e. the absolute pressure divided by the fluid density), $\boldsymbol{I}$ the identity matrix and $\varepsilon(\boldsymbol{U})$ the rate-of-strain tensor. The mass balance equations and their associated boundary conditions are given by Eqs. (3) below. It is important to point out that the reaction takes place only in the bulk of the reactor, i.e. in $\Omega$ and not on the walls $\Gamma$ and $\Gamma_{\text {lat }}$.

$$
\begin{aligned}
-D \Delta C+\boldsymbol{U} \cdot \nabla C+k C & =0 & & \text { in } \Omega \\
C & =C_{\text {in }} & & \text { on } \Gamma_{\text {in }} \\
\frac{\partial C}{\partial n} & =0 & & \text { on } \Gamma_{\text {lat }} \cup \Gamma_{\text {out }} \cup \Gamma
\end{aligned}
$$

where $C$ denotes the reactant concentration in $\Omega, k>0$ the reaction rate constant and $D>0$ the constant diffusion coefficient of the reactant. 


\subsection{Shape optimization problem formulation}

In what follows, we will assume, even at the cost of regularizing the boundary of the reactor, that $\Gamma_{\text {in }}, \Gamma_{\text {lat }}$ and $\Gamma_{\text {out }}$ denote fixed boundaries such that

- $\Gamma_{\text {in }}, \Gamma_{\text {lat }}$ and $\Gamma_{\text {out }}$ are respectively of positive 1-dimensional Hausdorff measure and their intersection two by two is of zero measure;

- $\Gamma_{\text {in }} \cup \Gamma_{\text {lat }} \cup \Gamma_{\text {out }}$ is the $\mathcal{C}^{1,1}$ (closed) boundary of a bounded connected domain standing for the external boundary of the reactor.

Formulating of the shape optimization problem requires the definition of a performance index, decisions variables and constraints, which are introduced below.

Performance index The objective here is to determine the shape of the fixed-bed reactor (i.e. the position and shape of the free boundary $\Gamma$ ) which maximizes the reaction conversion rate or minimizes the average outlet concentration of the reactant. The performance index is therefore defined by

$$
J(\Omega)=\int_{\Gamma_{\text {out }}} C d \sigma
$$

where $C$ denotes the solution of (3).

Decision variable The decision variable is defined by the free boundary $\Gamma$ that will evolve with the iterations of the optimization algorithm. The other boundaries are fixed.

Admissible set of constraints The optimization problem is subjected to different constraints, among which the process model equations (1)-(3). Let $\Omega_{0}$ be a reference structure for the fixed-bed reactor (typically, the one actually designed in the industry we aim at optimizing) assumed to have a regular boundary, say $\mathcal{C}^{1,1}$ (which will be commented in what follows). The other constraints consist of :

- an iso-volume constraint introduced in order to guarantee that residence times of admissible shapes have the same order of magnitude. This constraint reads

$$
H(\Omega)=0 \quad \text { where } \quad H(\Omega)=|\Omega|-\left|\Omega_{0}\right|,
$$

where $|\Omega|$ denotes as usually the Lebesgue measure of $\Omega$ (its surface in $2 \mathrm{D}$ ).

- an inequality constraint on the energy dissipation by the fluid due to viscous friction and is given by

$$
G(\Omega) \leqslant 0 \quad \text { where } \quad G(\Omega)=2 \nu \int_{\Omega}|\varepsilon(\boldsymbol{U})|^{2} d x-\underbrace{2 \nu \int_{\Omega_{0}}|\varepsilon(\boldsymbol{U})|^{2} d x}_{\mathcal{E}_{0}},
$$

where $(\boldsymbol{U}, p)$ denotes the solution of (1). Recall that the energy dissipation and the pressure drops are directly correlated. Therefore, such a constraint is necessary since it reflects the fact that the fluid must flow easily through the optimal geometry. 
- A regularity assumption: we assume the boundary of $\Omega$ regular enough, namely

$$
\Omega \text { has a } \mathrm{e}^{1,1} \text { boundary, }
$$

so that the solution of the systems (1)-(3) will be defined in a strong sense. This will in particular legitimate the computations that will be done in the sequel, including the use of Green formulas, the definition of the optimization criteria and so on.

- A geometric constraint: we will assume that

$$
\text { the considered domain } \Omega \text { standing for the reactor is connected }
$$

and that

$$
\text { its boundary contains } \Gamma_{i n}, \Gamma_{l a t} \text { and } \Gamma_{\text {out }} \text {. }
$$

As a conclusion, the first version of the optimal design problem is formulated below Considering only a geometrical constraint on the volume, and one on the energy dissipated by the fluid leads to the problem:

$$
\inf _{\Omega \in \mathcal{C}} J(\Omega)
$$

where

$$
\mathcal{C}:=\left\{\Omega \subset \mathbb{R}^{2} \mid \Omega \text { satisfies }(7)-(8)-(9), H(\Omega)=0 \text { and } G(\Omega) \leqslant 0\right\} .
$$

The numerical results for this problem will reveal that pieces of the optimized geometry cannot be easily manufactured by means of the additive manufacturing techniques considered in this work. For this reason, we will propose in Section 4 another version of this optimal design problem where 3D printing constraints are taken into account in order to enable the manufacturing of the computed optimal shape of the reactor.

Remark 1. An important question in the study of such problems is that of the existence of optimal shape(s). Investigating existences issues for general shape optimization problems involving fluid mechanics models is, up to our knowledge, open. Nevertheless, it is likely that imposing additional strong geometrical constraints such as an uniform cone property, will enforce existence (see e.g. $[8,9,24]$ ). Notice also that a similar constraint, unfortunately rather difficult to handle but nevertheless very interesting from the applicative point of view, will also be considered in Section 4.2.

\section{Optimization algorithm and numerical results}

\subsection{Computation of the shape derivative}

The numerical resolution of a shape optimization problem of the form $\left(\mathcal{P}_{1}\right)$ is based on the knowledge of the derivative of the optimized functional with respect to the variable domain. This notion can be understood within a variety of frameworks, one of them being the Hadamard boundary variation method which we presently sketch briefly (see e.g. [1, 23, 31, 34] for additional explanations).

In the setting of the Hadamard method, variations of a domain $\Omega$ with $\mathrm{e}^{1,1}$ boundary are considered under the form

$$
\Omega_{\boldsymbol{\theta}}=(\mathrm{Id}+\boldsymbol{\theta})(\Omega)
$$


where $\boldsymbol{\theta} \in W^{2, \infty}\left(\mathbb{R}^{2}, \mathbb{R}^{2}\right)$ is a 'small' vector field ${ }^{2}$ and Id $: \mathbb{R}^{2} \rightarrow \mathbb{R}^{2}$ is the identity mapping.

A shape functional $\Omega \mapsto J(\Omega)$ is said to be shape differentiable at $\Omega$ (in the sense of Hadamard) whenever the underlying mapping

$$
W^{2, \infty}\left(\mathbb{R}^{2}, \mathbb{R}^{2}\right) \ni \boldsymbol{\theta} \mapsto J\left(\Omega_{\boldsymbol{\theta}}\right) \in \mathbb{R}
$$

is Fréchet differentiable at $\boldsymbol{\theta}=0$. The corresponding derivative $J^{\prime}(\Omega)(\boldsymbol{\theta})$ is the so-called shape derivative of $J$ at $\Omega$ and the following expansion holds:

$$
J\left(\Omega_{\boldsymbol{\theta}}\right)=J(\Omega)+J^{\prime}(\Omega)(\boldsymbol{\theta})+\mathrm{o}(\boldsymbol{\theta}), \quad \text { where } \frac{\mathrm{o}(\boldsymbol{\theta})}{\|\boldsymbol{\theta}\|_{W^{2, \infty}\left(\mathbb{R}^{2}, \mathbb{R}^{2}\right)} \underset{\boldsymbol{\theta} \rightarrow 0}{\longrightarrow} 0 .}
$$

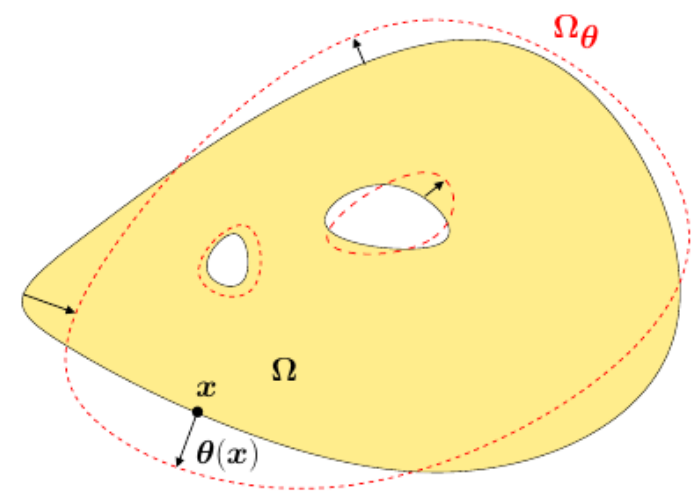

Figure 2: Example of admissible deformation. The domain $\Omega$ is plotted with a solid line and $\Omega_{\boldsymbol{\theta}}$ is plotted with a dotted line. $\boldsymbol{\theta}(\boldsymbol{x})$ represents the boundary variation at point $\boldsymbol{x} \in \partial \Omega$.

Before computing the shape derivative of $J$ at $\Omega$, let us recall that (1) and (3) have to be understood through their variational form. Let $\Omega$ denote a given element of $\mathcal{C}$. We introduce the functional spaces

$$
\begin{aligned}
\mathcal{U} & =\left\{(\boldsymbol{U}, p) \in H^{2}\left(\Omega, \mathbb{R}^{2}\right) \times H^{1}(\Omega, \mathbb{R}) \mid \operatorname{div} \boldsymbol{U}=0 \text { in } \Omega, \boldsymbol{U}=0 \text { on } \Gamma \text { and } \boldsymbol{U}=\boldsymbol{U}_{\text {in }} \text { on } \Gamma_{i n}\right\}, \\
\mathcal{V} & =\left\{(\boldsymbol{w}, q) \in H^{2}\left(\Omega, \mathbb{R}^{2}\right) \times H^{1}(\Omega, \mathbb{R}) \mid \operatorname{div} \boldsymbol{w}=0 \text { in } \Omega \text { and } \boldsymbol{w}=0 \text { on } \Gamma_{i n} \cup \Gamma\right\}, \\
\mathcal{W} & =\left\{C \in H^{2}(\Omega, \mathbb{R}) \mid C=C_{0} \text { on } \Gamma_{i n}\right\}, \\
\mathcal{X} & =\left\{u \in H^{2}(\Omega, \mathbb{R}) \mid u=0 \text { on } \Gamma_{i n}\right\} .
\end{aligned}
$$

The variational formulation associated to (1) reads: find $(\boldsymbol{U}, p) \in \mathcal{U}$ such that

$$
\forall(\boldsymbol{w}, q) \in \mathcal{V}, \quad \int_{\Omega} \varepsilon(\boldsymbol{U}): \varepsilon(\boldsymbol{w})+(\boldsymbol{U} \cdot \nabla \boldsymbol{U}) \boldsymbol{w}-q \operatorname{div} \boldsymbol{U}=0
$$

and the variational formulation associated to (3) reads: find $C \in \mathcal{W}$ such that

$$
\forall u \in X, \quad \int_{\Omega}(D \nabla C \cdot \nabla u+(\boldsymbol{U} \cdot \nabla C) u+k C u)=0 .
$$

\footnotetext{
${ }^{2}$ More precisely, the smallness assumption on $\theta$ can be understood as follows: one can prove that if $\|\boldsymbol{\theta}\|_{W^{2, \infty}\left(\mathbb{R}^{2}, \mathbb{R}^{2}\right)}<1$, then $\mathrm{Id}+\boldsymbol{\theta}$ is a diffeomorphism and moreover, $(\mathrm{Id}+\boldsymbol{\theta})^{-1}-\mathrm{Id}$ belongs to $W^{2, \infty}\left(\mathbb{R}^{2}, \mathbb{R}^{2}\right)$.
} 
The following well-posed results for the solutions of the PDEs (1) and (3) is rather standard. We refer for instance to $[20,35]$ for the fluid system, and more specifically to $[27,11]$ and $[21$, Chapter IX] for issues related to the regularity of the solutions. We also refer to $[10,17,33]$ for the convection-diffusion equation. A very close system is also investigated in [19]. The following theorem sums-up the well-posed character of the coupled system (1)-(3).

Theorem 1. Let $\Gamma$ be nonempty, such that $\Omega \in \mathcal{C}$. Let $\boldsymbol{U}_{\text {in }} \in H^{3 / 2}\left(\partial \Omega, \mathbb{R}^{2}\right)$ and $C_{0} \in H^{3 / 2}(\partial \Omega, \mathbb{R})$. If the viscosity $\nu$ is assumed to be large enough, then System (1)-(3) has a unique solution $(\boldsymbol{U}, p)$ in U. Similarly, under these conditions, the problem (3) has a unique solution $C$ in $\mathcal{W}$.

Let us now compute the shape derivative of $J$. The shape differentiability of $J$ is a standard issue, which is well referenced in the dedicated literature and has been much studied. Regarding the case we investigate, we claim that a standard approach, as described in [23, Chap. 5] would allow us to answer positively to this issue. The key point is to start from the variational formulation associated to the PDE problem on the deformed domain $\Omega_{\boldsymbol{\theta}}$, to use a change of variable to rewrite all the involved integrals on a fixed domain, and finally to use adequately the implicit function theorem to get the shape differentiability of the solutions of the PDEs under consideration. Very close differentiability studies have been led in $[19,9]$. Let us first formally introduce the tools for computing the shape derivatives.

Let $\Omega \in \mathcal{C}$ and $\boldsymbol{\theta} \in W^{2, \infty}\left(\mathbb{R}^{2}, \mathbb{R}^{2}\right)$ be a vector field whose support does not intersect $\Gamma_{\text {in }}, \Gamma_{\text {lat }}$ and $\Gamma_{\text {out }}$. Let us compute $J^{\prime}(\Omega)(\boldsymbol{\theta})$ defined by $(12)$. By following $\left.[1,23,14]\right)$, one gets

$$
J^{\prime}(\Omega)(\boldsymbol{\theta})=\left.\frac{d}{d t} \int_{\Gamma_{\text {out }}} C_{t} d \sigma\right|_{t=0}=\int_{\Gamma_{\text {out }}} C^{\prime} d \sigma
$$

where $C_{t}$ denotes the solution of (3) on the domain $(\operatorname{Id}+t \boldsymbol{\theta})(\Omega), \boldsymbol{U}^{\prime}, p^{\prime}$ and $C^{\prime}$ denote respectively the so-called Eulerian derivatives of $\boldsymbol{U}, p$ and $C$. From a qualitative point of view, $\boldsymbol{U}^{\prime}$ (resp. $p^{\prime}$ and $C^{\prime}$ ) stands for the sensitivity of $\boldsymbol{U}$ (resp. $p$ and $C$ ) with respect to shape variations. Standard differentiation formulas (see $[29,15,24])$ enable us to show that $\left(\boldsymbol{U}^{\prime}, p^{\prime}\right) \in \mathcal{V}$ solves (in a variational sense) the system

$$
\begin{aligned}
-\nu \Delta \boldsymbol{U}^{\prime}+\left(\boldsymbol{U}^{\prime} \cdot \nabla\right) \boldsymbol{U}+(\boldsymbol{U} \cdot \nabla) \boldsymbol{U}^{\prime}+\nabla p^{\prime} & =0 & & \text { in } \Omega \\
\nabla \cdot \boldsymbol{U}^{\prime} & =0 & & \text { in } \Omega \\
\boldsymbol{U}^{\prime} & =0 & & \text { on } \Gamma_{\text {in }} \cup \Gamma_{\text {lat }} \\
\boldsymbol{U}^{\prime} & =-\frac{\partial \boldsymbol{U}}{\partial n}(\boldsymbol{\theta} \cdot \boldsymbol{n}) & & \text { on } \Gamma \\
\boldsymbol{\sigma}\left(\boldsymbol{U}^{\prime}, p^{\prime}\right) \boldsymbol{n} & =0 & & \text { on } \Gamma_{\text {out }}
\end{aligned}
$$

Similarly, $C^{\prime}$ stands for the sensitivity of $C$ with respect to shape variations of $\Omega$ and $C^{\prime}$ solves in a variational sense the system

$$
\begin{aligned}
-D \Delta C^{\prime}+\boldsymbol{U} \cdot \nabla C^{\prime}+\boldsymbol{U}^{\prime} \cdot \nabla C+k C^{\prime} & =0 & & \text { in } \Omega \\
C^{\prime} & =0 & & \text { on } \Gamma_{\text {in }} \\
\frac{\partial C^{\prime}}{\partial n} & =\mathcal{H}(C, \boldsymbol{\theta}) & & \text { on } \Gamma \\
\frac{\partial C^{\prime}}{\partial n} & =0 & & \text { on } \Gamma_{\text {out }} \cup \Gamma_{\text {lat }}
\end{aligned}
$$

where $\mathcal{H}(C, \boldsymbol{\theta})=-\frac{\partial^{2} C}{\partial n^{2}}(\boldsymbol{\theta} \cdot \boldsymbol{n})+\nabla C \cdot(\nabla(\boldsymbol{\theta} \cdot \boldsymbol{n})-(\nabla(\boldsymbol{\theta} \cdot \boldsymbol{n}) \cdot \boldsymbol{n}) \boldsymbol{n})$. 
Unfortunately, the formula (13) of the shape derivative is not very exploitable as it is. To overcome this problem, it is relevant to rewrite it as an integral on the free boundary. For this purpose, let us introduce the adjoint variables $C_{a}$ as the solution of the diffusion system

$$
\begin{aligned}
-D \Delta C_{a}-\boldsymbol{U} \cdot \nabla C_{a}+k C_{a} & =0 & & \text { in } \Omega \\
C_{a} & =0 & & \text { on } \Gamma_{\text {in }} \\
\frac{\partial C_{a}}{\partial n} & =0 & & \text { on } \Gamma_{\text {lat }} \cup \Gamma \\
C_{a}(\boldsymbol{U} \cdot \boldsymbol{n})+D \frac{\partial C_{a}}{\partial n} & =1 & & \text { on } \Gamma_{\text {out }}
\end{aligned}
$$

and the variables $\left(\boldsymbol{U}_{a}, p_{a}\right)$ as the solution of the linearized Navier-Stokes system

$$
\begin{aligned}
-\nu \Delta \boldsymbol{U}_{\boldsymbol{a}}+(\nabla \boldsymbol{U})^{\top} \boldsymbol{U}_{\boldsymbol{a}}-\nabla \boldsymbol{U}_{\boldsymbol{a}} \boldsymbol{U}+\nabla p_{a} & =-C_{a} \nabla C & & \text { in } \Omega \\
\nabla \cdot \boldsymbol{U}_{\boldsymbol{a}} & =0 & & \text { in } \Omega \\
\boldsymbol{U}_{\boldsymbol{a}} & =0 & & \text { on } \Gamma_{\text {in }} \cup \Gamma_{\text {lat }} \cup \Gamma \\
\boldsymbol{\sigma}\left(\boldsymbol{U}_{\boldsymbol{a}}, p_{a}\right) \boldsymbol{n}+(\boldsymbol{U} \cdot \boldsymbol{n}) \boldsymbol{U}_{\boldsymbol{a}} & =0 & & \text { on } \Gamma_{\text {out }}
\end{aligned}
$$

where $(\boldsymbol{U}, p, C)$ denotes the solution of the coupled system (1)-(3)

A workable writing of the shape derivative $J^{\prime}(\Omega)(\boldsymbol{\theta})$ is thus derived in the following result.

Theorem 2. Let $\Gamma$ be nonempty, such that $\Omega \in \mathcal{C}$. Under the same assumptions on $\boldsymbol{U}_{0}$ and $C_{0}$ as in Theorem 1, if the viscosity $\nu$ is assumed to be large enough, then System (16)-(17) has a unique solution $\left(C_{a}, \boldsymbol{U}_{a}, p_{a}\right)$ in $X \times \mathcal{V}$.

Furthermore, let $\boldsymbol{\theta} \in W^{2, \infty}\left(\mathbb{R}^{2}, \mathbb{R}^{2}\right)$ be a vector field whose support does not intersect $\Gamma_{i n}, \Gamma_{\text {lat }}$ and $\Gamma_{\text {out }}$. Then there holds

$$
J^{\prime}(\Omega)(\boldsymbol{\theta})=\int_{\Gamma}\left(2 \nu \varepsilon(\boldsymbol{U}): \boldsymbol{\varepsilon}\left(\boldsymbol{U}_{\boldsymbol{a}}\right)-D C_{a} \Delta C\right)(\boldsymbol{\theta} \cdot \boldsymbol{n}) d \sigma .
$$

A proof of this result is provided in Appendix A.

\subsection{A Lagrangian approach}

The shape optimization approach developed hereafter is a gradient-based method which uses the socalled Hadamard boundary variation method [23] described in Section 3.1. To take the constraints into account in Problem $\left(\mathcal{P}_{1}\right)$, it is convenient to introduce the Lagrangian functional associated to the optimization problem, namely the functional $\mathcal{L}$ given by

$$
\mathcal{L}\left(\Omega, \lambda_{\mathcal{V}}, \lambda_{\mathcal{E}}\right)=K_{\text {crit }} J(\Omega)+\lambda_{\mathcal{V}} H(\Omega)+\lambda_{\mathcal{E}} G(\Omega),
$$

where $K_{\text {crit }}>0$ denotes a constant allowing to homogenize the Lagrangian functional (i.e. it ensures the same unit of measure between terms of Eq. (18)). $\lambda_{\mathcal{V}} \in \mathbb{R}$ and $\lambda_{\varepsilon} \in \mathbb{R}_{+}$stand for the Lagrange multipliers respectively associated to the constraints $H(\Omega)=0$ and $G(\Omega) \leqslant 0$.

Before providing the main idea of the algorithm, we need to compute the shape derivative of $\mathcal{L}$. Let $\boldsymbol{\theta} \in W^{2, \infty}\left(\mathbb{R}^{2}, \mathbb{R}^{2}\right)$ be a vector field whose support does not intersect $\Gamma_{\text {in }}, \Gamma_{\text {lat }}$ and $\Gamma_{\text {out }}$. We denote by $\mathcal{L}^{\prime}\left(\Omega, \lambda_{\mathcal{V}}, \lambda_{\mathcal{E}}\right)(\boldsymbol{\theta})$ the shape derivative of $\mathcal{L}$ (with respect to its first variable), in other words

$$
\mathcal{L}^{\prime}\left(\Omega, \lambda_{\mathcal{V}}, \lambda_{\mathcal{E}}\right)(\boldsymbol{\theta})=\lim _{t \searrow 0} \frac{\mathcal{L}\left(\Omega_{t}\right)-\mathcal{L}(\Omega)}{t}, \quad \text { with } \quad \Omega_{t}=(\operatorname{Id}+t \boldsymbol{\theta})(\Omega) .
$$


Let us introduce the adjoint state $\left(\boldsymbol{U}_{\boldsymbol{a}}, p_{a}\right)$ as the solution of the system

$$
\begin{aligned}
\mathcal{L}\left(\boldsymbol{U}_{\boldsymbol{a}}, \nabla p_{a}\right) & =-\lambda_{\varepsilon} 2 \nu \Delta \boldsymbol{U}-K_{\text {crit }} C_{a} \nabla C & & \text { in } \Omega \\
\nabla \cdot \boldsymbol{U}_{\boldsymbol{a}} & =0 & & \text { in } \Omega \\
\boldsymbol{U}_{\boldsymbol{a}} & =0 & & \text { on } \Gamma_{\text {in }} \cup \Gamma_{\text {lat }} \cup \Gamma \\
\boldsymbol{\sigma}\left(\boldsymbol{U}_{\boldsymbol{a}}, p_{a}\right) \boldsymbol{n}+(\boldsymbol{U} \cdot \boldsymbol{n}) \boldsymbol{U}_{\boldsymbol{a}} & =4 \nu \lambda_{\varepsilon} \varepsilon(\boldsymbol{U}) \boldsymbol{n} & & \text { on } \Gamma_{\text {out }}
\end{aligned}
$$

where $\mathcal{L}\left(\boldsymbol{U}_{\boldsymbol{a}}, \nabla p_{a}\right)=-\nu \Delta \boldsymbol{U}_{\boldsymbol{a}}+(\nabla \boldsymbol{U})^{\top} \boldsymbol{U}_{\boldsymbol{a}}-\nabla \boldsymbol{U}_{\boldsymbol{a}} \boldsymbol{U}+\nabla p_{a}$ and $C_{a}$ as the solution of the system

$$
\begin{aligned}
-D \Delta C_{a}-\boldsymbol{U} \cdot \nabla C_{a}+k C_{a} & =0 & & \text { in } \Omega \\
C_{a} & =0 & & \text { on } \Gamma_{\text {in }} \\
\frac{\partial C_{a}}{\partial n} & =0 & & \text { on } \Gamma_{\text {lat }} \cup \Gamma \\
C_{a}(\boldsymbol{U} \cdot \boldsymbol{n})+\mathcal{D} \frac{\partial C_{a}}{\partial n} & =1 & & \text { on } \Gamma_{\text {out }}
\end{aligned}
$$

Proposition 1. Under the same assumptions as in Theorem 2, the coupled system (20)-(19) has a unique solution $\left(C_{a}, \boldsymbol{U}_{a}, p_{a}\right)$ in $\mathcal{X} \times \mathcal{V}$ and there holds

$$
\begin{array}{r}
\mathcal{L}^{\prime}\left(\Omega, \lambda_{\mathcal{V}}, \lambda_{\varepsilon}\right)(\boldsymbol{\theta})= \\
\int_{\Omega}\left(2 \nu\left(\varepsilon(\boldsymbol{U}): \varepsilon\left(\boldsymbol{U}_{\boldsymbol{a}}\right)-\lambda_{\varepsilon} \varepsilon(\boldsymbol{U}): \varepsilon(\boldsymbol{U})\right)-K_{c r i t} D C_{a} \Delta C+\lambda_{\mathcal{V}}\right)(\boldsymbol{\theta} \cdot \boldsymbol{n}) d \sigma .
\end{array}
$$
by

It is notable that the shape gradient $g(\Omega)$, defined on the free boundary $\Gamma$, is therefore given

$$
g(\Omega)=2 \nu\left(\varepsilon(\boldsymbol{U}): \varepsilon\left(\boldsymbol{U}_{\boldsymbol{a}}\right)-\lambda_{\varepsilon} \varepsilon(\boldsymbol{U}): \varepsilon(\boldsymbol{U})\right)-K_{\text {crit }} D C_{a} \Delta C+\lambda_{\nu} .
$$

Its computation needs the knowledge of all the state and adjoint variables $(\boldsymbol{U}, p$ and $C)$ and $\left(\boldsymbol{U}_{a}\right.$, $p_{a}$ and $C_{a}$ ). From the numerical point of view, we will use the gradient to build an iterative optimization algorithm. In a nutshell, at each iteration, we will infer a displacement vector field $\boldsymbol{\theta}$ (a descent direction of the Lagrangian) from the knowledge of $g(\Omega)$ and modify adequately each node of the mesh.

Let us explain how to compute the vector field $\boldsymbol{\theta}_{\boldsymbol{i}}$ At the iteration $i\left(i \in \mathbb{N}^{*}\right)$. Consider the domain $\Omega_{i}$ obtained after $i$ iterations of the algorithm. The vector field $\boldsymbol{\theta}_{\boldsymbol{i}}$ is determined in such a way that

$$
\mathcal{L}^{\prime}\left(\Omega, \lambda_{\mathcal{V}}, \lambda_{\mathcal{E}}\right)\left(\boldsymbol{\theta}_{\boldsymbol{i}}\right)=-\gamma\left\|\nabla \boldsymbol{\theta}_{\boldsymbol{i}}\right\|_{L^{2}\left(\Omega_{i}\right)}^{2}-\left\|\boldsymbol{\theta}_{\boldsymbol{i}}\right\|_{L^{2}\left(\Omega_{i}\right)}^{2} .
$$

Observe that $\gamma\|\nabla \cdot\|_{L^{2}\left(\Omega_{i}\right)}^{2}+\|\cdot\|_{L^{2}\left(\Omega_{i}\right)}^{2}$ defines a norm equivalent to the standard $H^{1}$ norm. The introduction of the parameter $\gamma$ will allow us to adjust the diffusivity strength on the mesh.

The choice of this modified $H^{1}$ norm is motivated by the willing of getting a smooth enough vector field. Such an issue has been much discussed in [16] and yields to defining $\boldsymbol{\theta}_{\boldsymbol{i}}$ as the unique solution (in a weak variational sense) of the PDE

$$
\begin{aligned}
-\gamma \Delta \boldsymbol{\theta}+\boldsymbol{\theta} & =0 & & \text { in } \Omega \\
\boldsymbol{\theta} & =0 & & \text { on } \Gamma_{\text {in }} \cup \Gamma_{\text {out }} \cup \Gamma_{\text {lat }} \\
\gamma \nabla \boldsymbol{\theta} \boldsymbol{n} & =-g \boldsymbol{n} & & \text { on } \Gamma
\end{aligned}
$$

Once the vector field $\boldsymbol{\theta}$ computed, we then move all mesh-nodes according to

$$
\Omega_{i+1}=\left(\operatorname{Id}+t_{i} \boldsymbol{\theta}_{\boldsymbol{i}}\right)\left(\Omega_{i}\right)
$$

where $t_{i}>0$ is the method step which must be chosen adequately (for instance with the help of a 1D optimization method), Id is the identity operator. 


\subsection{Optimization algorithm}

The numerical optimization problem presented in this work is implemented within OpenFOAM environment which is a free and open source software package [37]. This CFD software solves numerically a large range of PDEs using the finite volume method. In our case, OpenFOAM solves the system of Navier-Stokes equations (1) combined with the mass balance equations (3), the adjoint system equations (16)-(17)) and computes the mesh diffusion displacement through (22). The Python library named "pyFoam " is used in order to connect optimization iterations to each other using its utility "pyFoamMeshUtilityRunner.py". The developed algorithm consists of the folowing steps:

1. Meshing of the initial shape using cfMesh and snappyHexMesh, two mesh utilities supplied by OpenFOAM. The resulting mesh is composed of 50,000 to 70,000 meshes whose sizes are between $2 \times 10^{-4} \mathrm{~m}$ and $9 \times 10^{-4} \mathrm{~m}$. About $90 \%$ of the meshes are quadrilaterals with the remaining $10 \%$ being composed of triangular meshes (Fig. 3).

According to (21), the shape gradient functional is defined on the free boundary and depends on the first and second order derivatives of the state and adjoint variables. Therefore, all these quantities need to be computed with accuracy near wall zones, and we therefore add 2 layers of mesh near the boundary $\Gamma$.

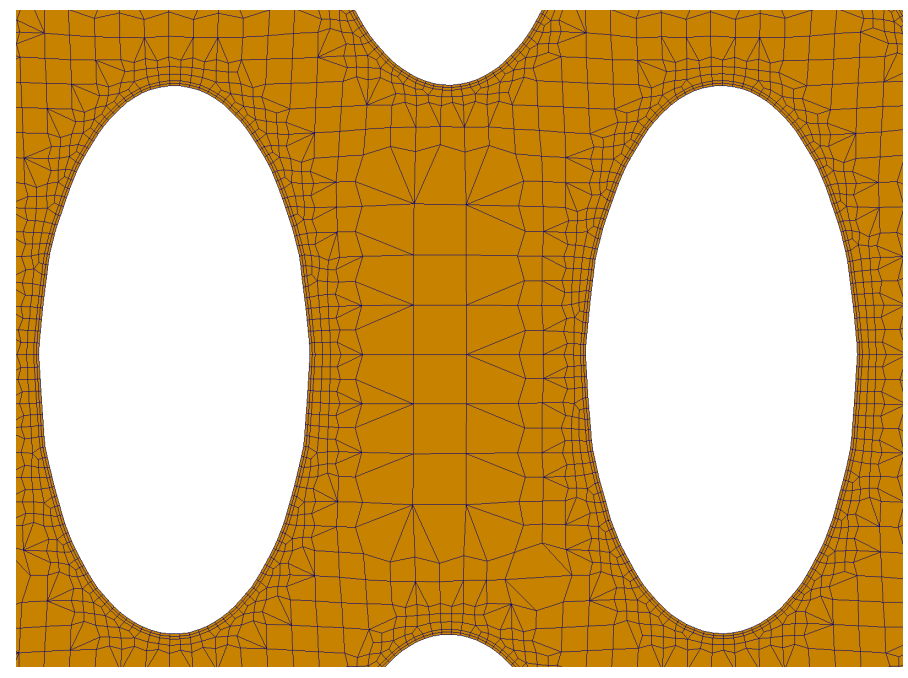

Figure 3: Exemple of the generated mesh.

2. Resolution of the process model equations (1)-(3) and the adjoint system equations (16)(17). The SIMPLE algorithm is used in order to deal with the velocity-pressure coupling of Eqs. (1)-(17). The convergence of the CFD algorithm is carried out through the nomarlized residual of the discretized PDEs [30]. If it is lower than $10^{-5}$, the CFD convergence is considered as achieved.

3. The shape gradient $g(\Omega)$ and the mesh diffusion $\boldsymbol{\theta}$ are computed. The obstacle and channel constraints are taken into account during this step (see $\S 4.2)$. 
4. Update of the Lagrange multipliers at the $k$-th iteration according to the following relations:

$$
\begin{aligned}
& \lambda_{\mathcal{V}}^{k+1}=\lambda_{\mathcal{V}}^{k}+\beta_{\mathcal{V}} H(\Omega) \\
& \lambda_{\mathcal{E}}^{k+1}=\max \left(0, \lambda_{\mathcal{E}}^{k}+\beta_{\mathcal{E}} G(\Omega)\right)
\end{aligned}
$$

In these equations, $\beta_{\mathcal{V}}$ and $\beta_{\mathcal{E}}$ denote small positive parameters.

5. At the end of iterations, a mesh quality test using three criteria is performed and a test on the convergence is carried out. Fig. 4 illustrates the mesh quality criteria. According to [26], the criteria are:

- the mesh aspect ratio defined as the ratio of the longest side over the shortest one among all cells of the mesh, namely $r=l / s$ on Fig. 4 .

- the mesh non-orthogonality which corresponds to the angle between the line linking two adjacent cell centers and the normal to their common face, namely the angle $\alpha=$ $\arccos \left(\frac{\boldsymbol{A}_{i} \cdot \boldsymbol{C}_{\boldsymbol{i}}}{\left|\boldsymbol{A}_{\boldsymbol{i}} \| \boldsymbol{C}_{\boldsymbol{i}}\right|}\right)$ on Fig. 4.

- the face skewness which is the ratio of the distance $\left|\boldsymbol{d}_{\boldsymbol{i}}\right|$ over the distance $\left|\boldsymbol{C}_{\boldsymbol{i}}\right|$, where $\left|\boldsymbol{d}_{\boldsymbol{i}}\right|$ denotes the distance between the intersection of the line linking two adjacent cell centers with their common face and their common face center, and $\left|\boldsymbol{C}_{\boldsymbol{i}}\right|$ denotes the distance between the two considered cell centers.

The remeshing process takes place whenever the aforementioned criteria are greater than 10, 65 and 3.8 respectively. From a practical point of view, we have observed that the remeshing operation is performed on average every 5 iterations. The convergence criterion of the algorithm is the ratio of the standard deviation of the 50 last Lagrangian values over the average of these 50 values. If the ratio is less than $10^{-4}$, the algorithm stops.

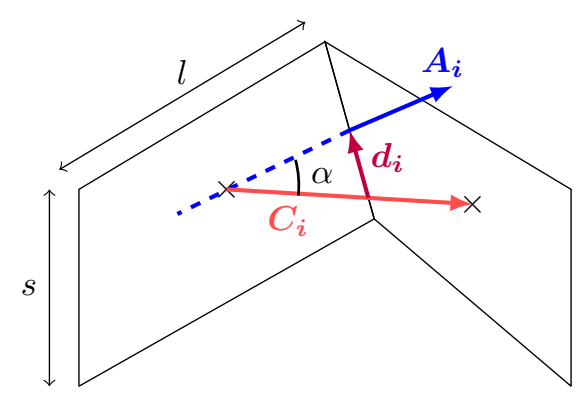

Figure 4: Illustration of the mesh quality criteria.

\section{Numerical results and discussion}

This section is devoted to the presentation of the numerical results obtained by implementing the algorithm described in Section 3.3. We also enrich our model by introducing a modified version of the shape optimization problem $\left(\mathcal{P}_{1}\right)$, taking into account several manufacturing constraints. In both cases, the numerical parameters used for the simulations are summarized in Table 1 . In the following sections, we introduce the new criteria we will deal with, as well as a simple optimization algorithm. 
Table 1: Parameters used for the simulations.

\begin{tabular}{ccc}
\hline Parameters & Values & Units \\
\hline$\nu$ & $10^{-6}$ & $\mathrm{~m}^{2} \cdot \mathrm{s}^{-1}$ \\
$U_{\text {in }}$ & $10^{-2}$ & $\mathrm{~m}^{-1} \mathrm{~s}^{-1}$ \\
$\mathcal{D}$ & $10^{-9}$ & $\mathrm{~m}^{2} \cdot \mathrm{s}^{-1}$ \\
$k$ & $10^{-2}$ & $\mathrm{~s}^{-1}$ \\
$\gamma$ & $10^{-4}$ & $\mathrm{~m}^{-2}$ \\
$K_{\text {crit }}$ & $3 \times 10^{-5}$ & $\mathrm{~m}^{6} \cdot \mathrm{s}^{-3} \cdot \mathrm{mol}^{-1}$ \\
$\beta_{\mathcal{V}}$ & $3 \times 10^{-5}$ & - \\
$\beta_{\mathcal{E}}$ & $3 \times 10^{-5}$ & - \\
$\mathrm{t}$ & 20 & - \\
\hline
\end{tabular}

\subsection{Numerical solution of Problem $\left(\mathcal{P}_{1}\right)$}

Figure 5 illustrates the concentration profiles of the reactant for : (a) the initial design of the reactor, (b) optimal shapes for Problem $\left(\mathcal{P}_{1}\right)$, by following to the algorithm described in Section 3.3.
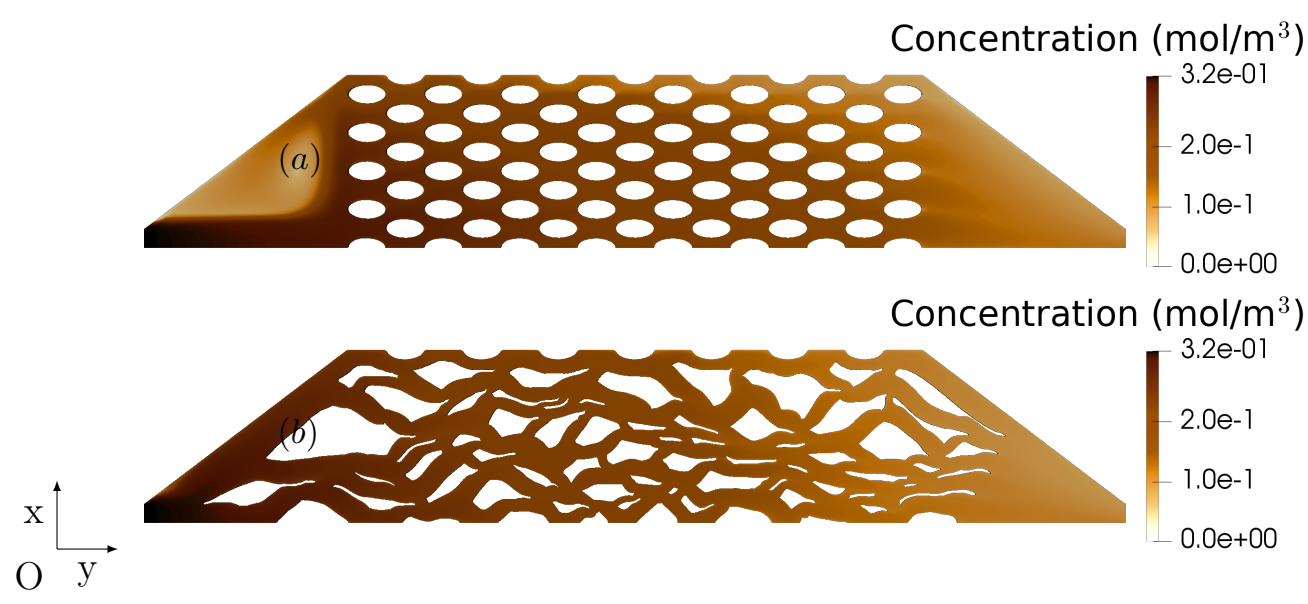

Figure 5: Initial configuration of the fixed-bed reactor (a), optimized shape without manufacturing constraint (b).

As can be seen, a dead zone appears at the entrance to the reactor of the initial configuration. This dead zone is a region where the reactant concentration is low, which produces a low reaction rate (recall that the conversion rate is proportional to the reactant concentration $r=k C$ ). As a result, this reactor dead volume is almost useless.

To illustrate the effectiveness of the method, we provide on Fig. 6 the convergence curves related to this shape optimization problem, namely the evolution of the Lagrangian functional, the performance index and the two constraints $H$ and $G$ with respect to the iteration number. According to Fig. 6 , the performance index has decreased by almost $10 \%$ which will improve the conversion rate accordingly. The volume and energy dissipation constraints are satisfied and it is interesting to point out that the energy inequality constraint is active. 

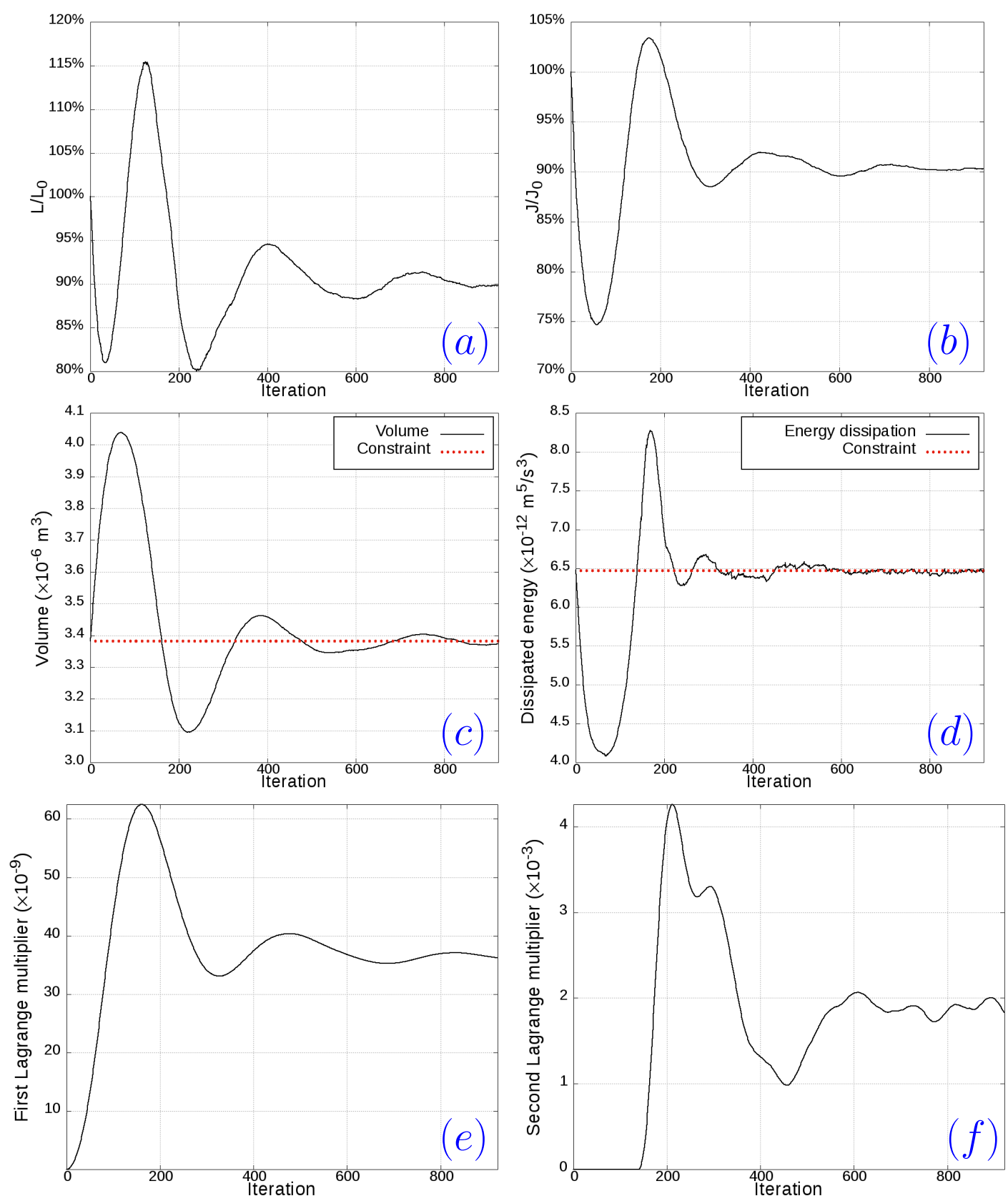

Figure 6: Convergence curves of the optimization process, $(a)$ is the ratio $\frac{\mathcal{L}}{\mathcal{L}_{0}},(b)$ the ratio $\frac{J}{J_{0}},(c)$ the volume constraint, $(d)$ the energy disspation constraint, $(e)$ the Lagrange multiplier associated to the volume constraint and $(f)$ the Lagrange multiplier associated to the energy constraint over iterations.

However, these first results are unfortunately not directly usable for practical purposes if fixedbed reactors are to be built by means of an additive manufacturing technique. Indeed, the Stratoconception process used to build the reactor requires minimal width on channels and obstacles 
making the process and the designed object robust. In the following sections, we slightly modify our approach to take into account the manufacturing constraints.

\subsection{New version of the optimization problem: introduction of manufac- turing constraints}

Since the resulting optimal shape will be manufactured by means of a 3D printing technique, additional constraints should be accounted for in the optimization problem. The new optimization problem is obtained by adding these new constraints on the formulation $\left(\mathcal{P}_{1}\right)$.

They are of inequality type and impose minimum values on the pores width (domain $\Omega$ ) and on the packing "thickness". There are several ways of modeling such a constraint. Let us describe hereafter the chosen approach.

It is not straightforward to define and use minimum thickness constraints inside a Lagrangian functional. The manufacturing constraints taken into account during the mesh diffusion step modify the vector field $\boldsymbol{\theta}$ after computation. This approach corresponds to a projected gradient type method. The obstacle and channel constraints are not considered using the same algorithm. Let us provide hereafter some explanations about these facts.

The method considered in the present paper aims to improve an existing design to include the manufacturing constraints. Since the objective of this work is not centered on manufacturability issues, we propose in the following a rather naive approach to take into account manufacturing constraints in order to quickly obtain first results. The proposed treatment is perfectible and can be improved following the more advanced works [5, 4, 3, 2] on the subject, which cleverly includes these constraints within an algorithm using an appropriate dual version of the optimization problem.

\subsubsection{Obstacle minimum thickness constraint}

The approach we chose is based on the notion of reach of a set [18]. Let $h>0$. A closed set $A$ is said to have a reach larger than $h$ provided that every point $x$ belonging to $\left\{x \in \mathbb{R}^{2} \mid \operatorname{dist}(x, A)<h\right\}$ has a unique projection point on $A$. The supremum of such $h$ is called the reach of $A$, and denoted as reach $(A)$. We will impose a thickness constraint by imposing the constraint

$$
T(\Omega) \geqslant 0 \quad \text { where } \quad T(\Omega)=\operatorname{reach}\left(\Omega^{c}\right)-d_{\text {min }}^{\text {obstacle }},
$$

where $d_{\min }^{\text {obstacle }}>0$ denotes the minimal obstacle width constraint and $\Omega^{c}$ the complement set of $\Omega$ in the domain delimited by $\Gamma_{\text {in }}, \Gamma_{\text {out }}$ and $\Gamma_{\text {lat }}$ (i.e. the full domain without pores).

This obstacle constraint is taken into account in 2 main steps: the first one involves the construction of the skeleton of each obstacle defined as follows: for each obstacle, the skeleton is the set of points such that their distance to the boundary of the obstacle is achieved for at least two distinct points of the boundary.

The algorithm allowing us to construct the skeleton reads as follows:

1. The Voronoi diagram of the boundary points is computed. This diagram of a set of points $E$ is constructed from Voronoi regions. In two dimensions, given several generating points, these regions correspond to cells partitioning a plane into convex polygons such that each polygon contains exactly one generating point and every point in a given polygon is closer to its generating point than to any other [7].The mathematical definition is given in Definition 1 below.

Definition 1. Let $X$ a subset of $\mathbb{R}^{d}$ and $P=\left\{P_{1}, P_{2}, \ldots, P_{n}\right\} \subset X$ a set of points. The Voronoi region $R_{k}$ of the point $P_{k}$ is defined as

$$
R_{k}=\left\{x \in X \mid d\left(x, P_{k}\right)<d\left(x, P_{j}\right), \quad \forall j \neq k\right\}
$$


where $d\left(x, P_{k}\right)$ denotes the distance between $x$ and $P_{k}$.

Each edge of the Voronoi diagram is the boundary of 2 Voronoi regions and the intersections of edges, called vertices of the diagram, are the boundary of 3 (or more) Voronoi regions. Each edge is bounded by 2 vertices. The skeleton is constructed from the edges of the Voronoi diagram.

2. The Voronoi diagram (Fig. 7(a)) is then simplified using two steps in order to compute the skeleton.

- Only edges completely included in the obstacle are kept (Fig. 7(b)).

- A second simplification is carried our by means of 2 criteria. (i) For all Voronoi vertices $s$, the first criterion is the function $r(s)$ that associates the maximum disc radius centered in $s$. (ii) Each point $s$ has three (or more) projections on the obstacle, called $p_{1}, p_{2}$ and $p_{3}$, the second criterion is given by $\alpha(s)=\max \left(\widehat{p_{1} s p_{2}}, \widehat{p_{1} s p_{3}}, \widehat{p_{2} s p_{3}}\right)$. The lower bound of those criteria is respectively $2 \times 10^{-4} \mathrm{~m}$ and $\frac{\pi}{4}$ (Fig. 7 (c)).

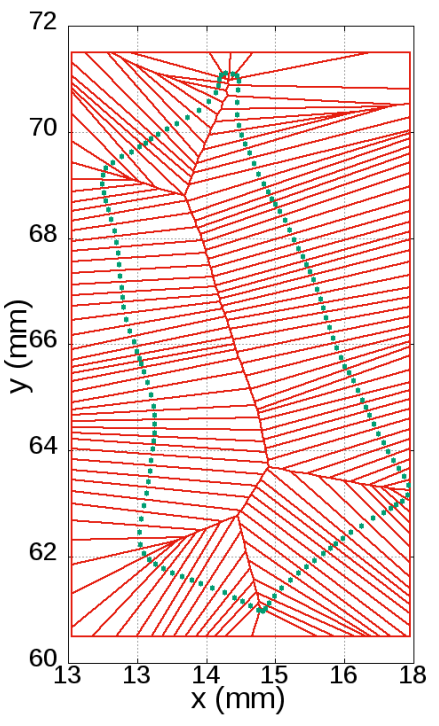

(a) Obstacle Voronoi diagram

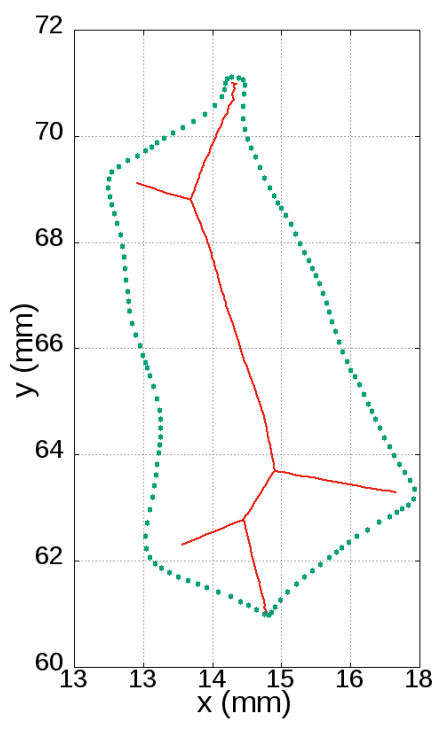

(b) First simplification

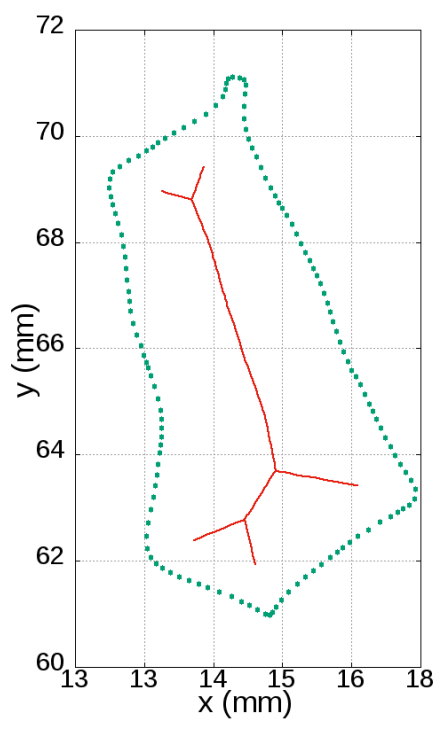

(c) Second simplification: skeleton

Figure 7: Simplification of the Voronoi diagram into the skeleton, green points denote obstacle points and red line denotes Voronoi diagram $(a)$ and the simplified skeleton $(b)$ and $(c)$. 


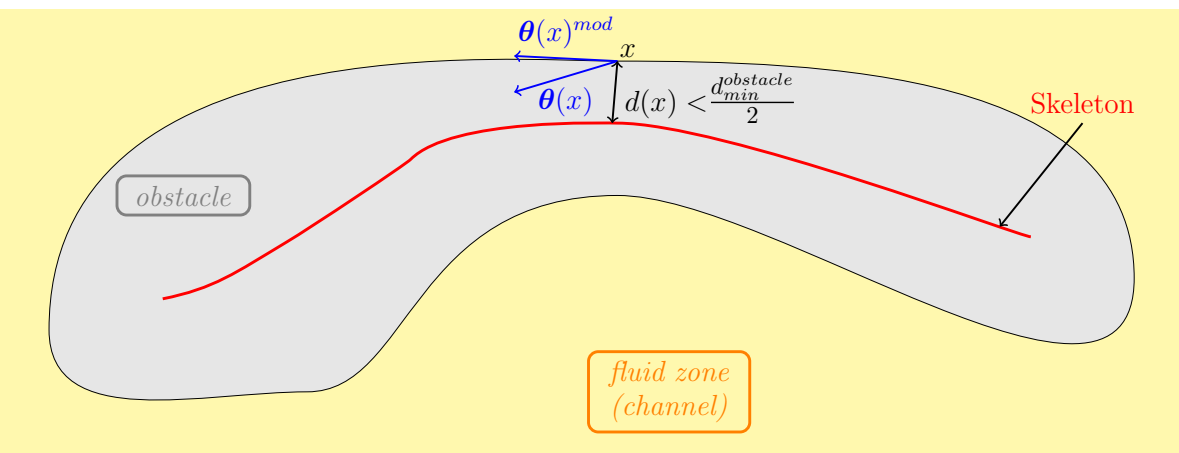

Figure 8: Illustration of the obstacle constraint consideration. $\boldsymbol{\theta}(x)$ and $\boldsymbol{\theta}(x)^{\text {mod }}$ represent respectively the mesh displacement at point $x$ before and after modification using the projection method.

Once the skeleton constructed, a test on the minimum distance between the obstacle and the skeleton named $d(x)$ is performed. If this distance $d(x)$ is lower than $d_{\min }^{\text {obstacle }} / 2$ and $d(x)>$ $d(x+t \boldsymbol{\theta}(x))$ then the vector $\boldsymbol{\theta}(x)$ is projected to the corresponding skeleton tangent vector (Fig. 8). The resulting projection is called $\boldsymbol{\theta}(x)^{\text {mod }}$.

\subsubsection{Channel minimum width constraint}

The channel constraints are taken into account as follows (see the illustration on Fig. 9):

1. For each boundary point $x$ of a given obstacle, determine the nearest boundary point $x^{\text {near }}$ belonging to another obstacle boundary.

2. We compute the inner product $\boldsymbol{x} \boldsymbol{x}^{\text {near }} \cdot \boldsymbol{\theta}(x)$. If $\boldsymbol{x} \boldsymbol{x}^{\text {near }} \cdot \boldsymbol{\theta}(x)>0$ and $\left\|\boldsymbol{x} \boldsymbol{x}^{\text {near }}\right\|<d_{\text {min }}^{\text {chan }}$ then the vector $\boldsymbol{\theta}(x)$ is projected on the orthogonal line to the vector $\boldsymbol{x} \boldsymbol{x}^{\boldsymbol{n} \boldsymbol{e a r}}$. The resulting vector is called $\boldsymbol{\theta}(x)^{\text {mod }}$.

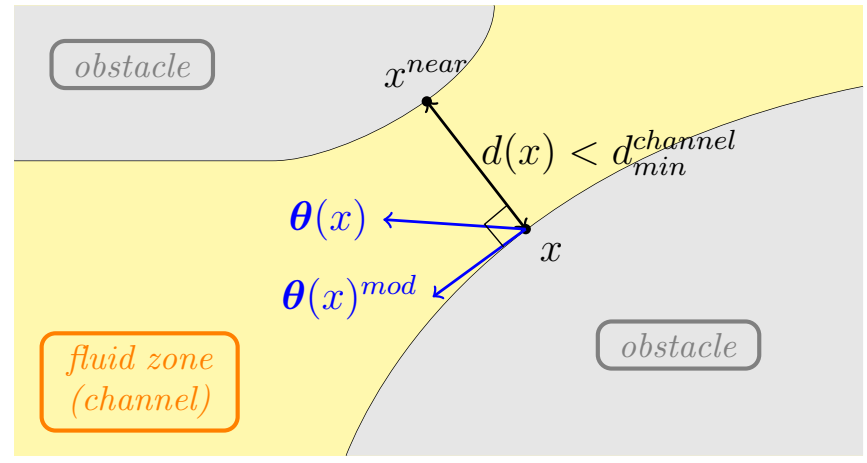

Figure 9: Illustration of the channel constraint treatment. $\boldsymbol{\theta}(x)$ and $\boldsymbol{\theta}(x)^{\text {mod }}$ represent respectively the mesh displacement at point $x$ before and after modification using the projection method. 


\subsection{Fixed-bed reactor optimization under energy, volume and manufac- turing constraints}

We investigate hereafter how is the optimal design modified whenever manufacturing constraints are added to the optimization algorithm in order to design the optimal reactor. The lower bounds of the obstacle thickness and the channel width imposed by the manufacturing process are respectively $2 \mathrm{~mm}$ and $2.1 \mathrm{~mm}$. On Figure 10(a), we provide the concentration profiles of the reactant in optimized shape when width constraints are integrated to the algorithm. Similarly to what was observed in optimal shape obtained for Problem $\left(\mathcal{P}_{1}\right)$ (without manufacturing constraints, see Fig. 1), no dead zone arises when taking into account the manufacturing constraints, even if stratoconception process used to build the reactor requires minimal width on channels and obstacles. The additional manufacturing constraints in the problem make optimization slightly harder since 200 more iterations are required to reach convergence.

The residence time distributions (RTD) of the initial and optimized designs under manufacturing constraints are presented on Fig. 11. In chemical engineering, the RTD is defined as the statistical time distribution that a set of particles stays in the reactor. It allows to characterize the reactor hydrodynamics. The RTD curves show a better homogeneity of the fluid flow in the optimized configuration (Fig. 10(b)) where the standard deviation is 3.5 times lower than in the initial configuration ( $21 \mathrm{~s}$ versus $73 \mathrm{~s}$ ). The reduction of the RTD standard deviation is mainly explained by the disappearance of the dead zone. Indeed, Fig. 11 shows that the RTD function reaches near zero values when evaluated for the optimal shape after $250 \mathrm{~s}$ while this is not the case for initial one. This better homogeneity can also be observed on Fig. 10 where the concentration gradient in the horizontal direction is much larger in the initial shape than in the optimized one. Finally, the disappearance of the dead region along with the better homogeneity of the flow improve the performance index by $9.8 \%$.

The impact of the initial reactor configuration on the optimal one has then been analyzed. On Fig. 10, we provide the numerical results starting the optimization process from slightly perturbed initial configurations: from a reactor where initial cylindrical obstacles half axis are increased by 0.5 $\mathrm{mm}$ (b), deacresed by $0.5 \mathrm{~mm}$ (c) and from a reactor where obstacles are moved by $3 \mathrm{~mm}$ towards the inlet (d) and towards the outlet (e). The optimal shapes displayed on Fig. 10 are slightly different from one another which shows the presence of local minima. However, the performance index is improved in the same proportions (between 9.7 and $9.8 \%$ ), therefore, none of these 5 local minima is much more effective than others.

The influence of the energy constraint has also been studied. The optimization process has been performed twice again increasing and decreasing the maximum value of the constraint by a factor 2 and the corresponding shapes are presented on Fig. 12. As we can see, the optimal shape (a) subject to low energy values constraint (i.e. maximum value of energy constraint divided by 2) presents larger fluid channels which allow a reduction of the velocity ensuring smaller velocity gradients and thus smaller energy dissipation by the fluid. However, the fluid flow in the reactor becomes inhomogeneous leading to a reduction of the criterion improvement by $7.5 \%$. When we increase the maximum energy value (b), smaller fluid channels become smaller which leads to an increase of the dissipated energy. However, in this case, it does not allow a significant decrease in the performance index compared to configurations displayed in Fig. 10 (9.9 versus 9.8\%). From an industrial point of view, shape (b) of the reactor is not relevant since it allows insignificant reduction of the performance index for a consequent increase in energy dissipation compared to shapes presented in Fig. 10. 

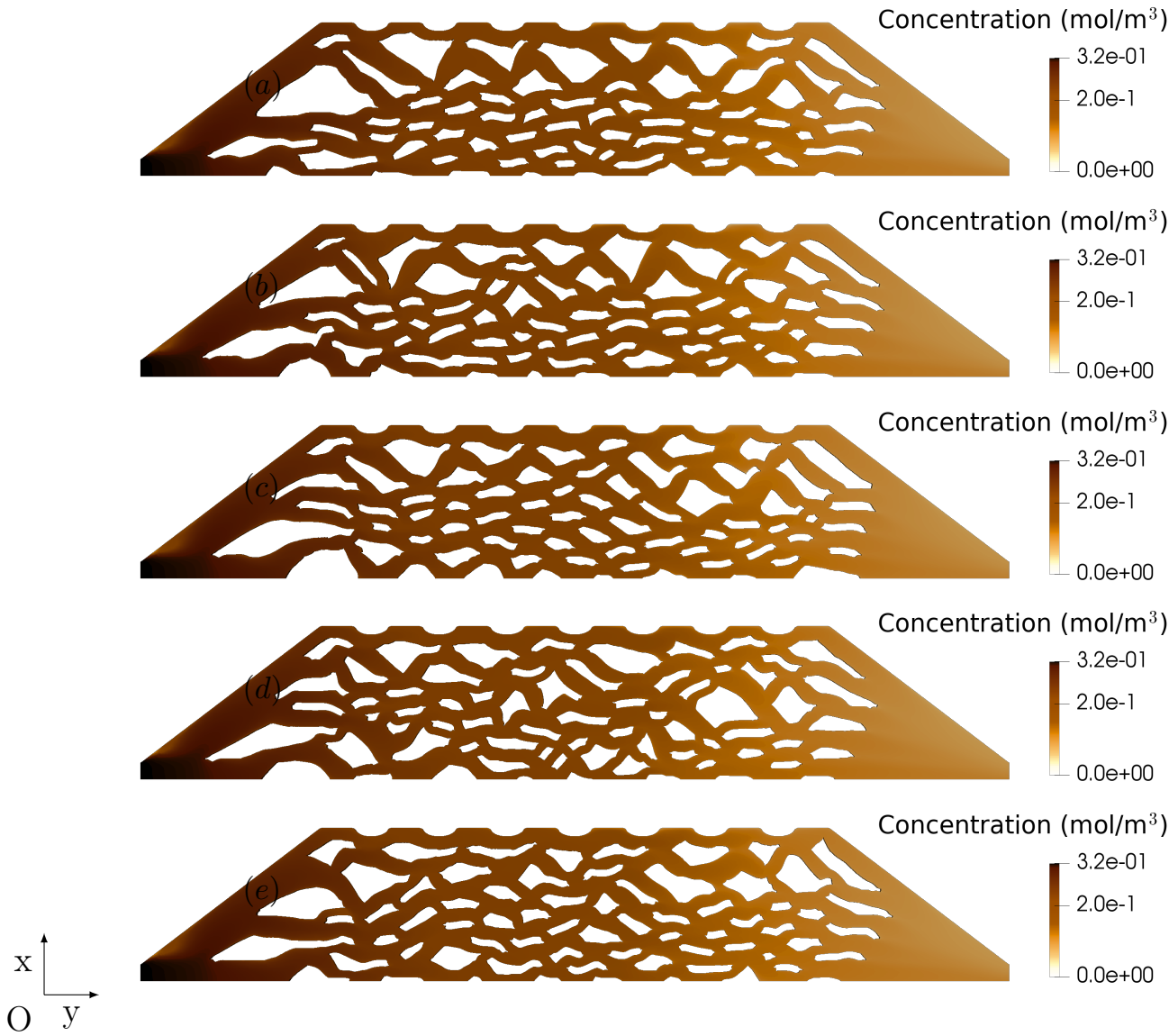

Figure 10: Optimized shapes with manufacturing constraints starting optimization process from, (a) reactor displayed on Fig. 1, (b) reactor where initial obstacles slightly bigger (half axis incresed by $0.5 \mathrm{~mm}$ ), (c) reactor where initial obstacles slightly smaller (half axis decreased by $0.5 \mathrm{~mm}$ ), (d) reactor where obstacles are translated by $3 \mathrm{~mm}$ towards the inlet and (e) reactor where obstacles are translated by $3 \mathrm{~mm}$ towards the outlet.

To finish, the topology of the domain is studied. Figure 13 presents the numerical result of the reactor optimization starting the process from a configuration with 72 holes instead of 128 . In order to compare optimal shapes the values associated to the volume and energy constraints are identical to those of Problem $\left(\mathcal{P}_{1}\right)$. In this case, the improvement of the performance index is lower (only by $7.9 \%$ with respect to intial configuration of the reactor with 128 holes 1 ) than for a configuration with 128 holes, which leads us to conclude that the topology of the domain influences the reactor performances. In this case, the number of holes is not large enough to allow the same quality of fluid flow homogenization in the reactor.

\section{Conclusion and perspectives}

In this paper, we have developed a geometric shape optimization approach for the design and sizing of processes. We are more specifically interested in the shape of the packing of a fixed- 


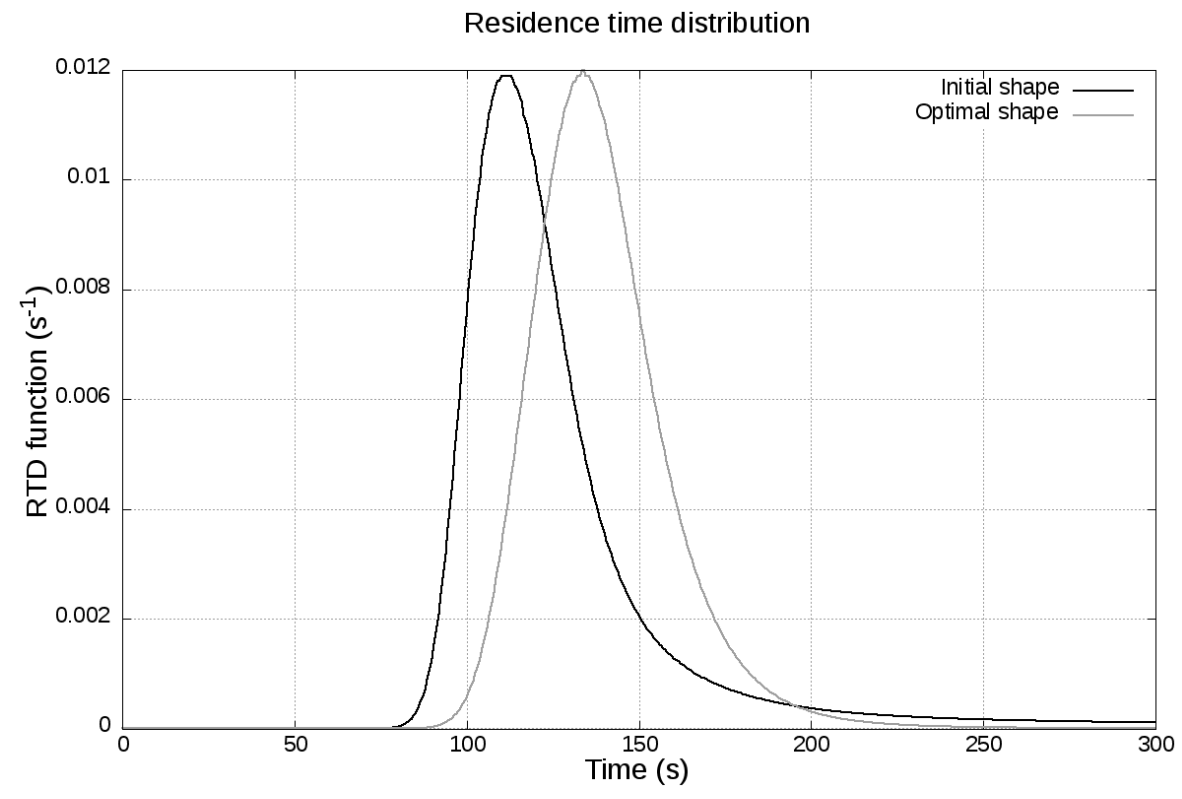

Figure 11: Residence time distribution of initial and optimized shapes.

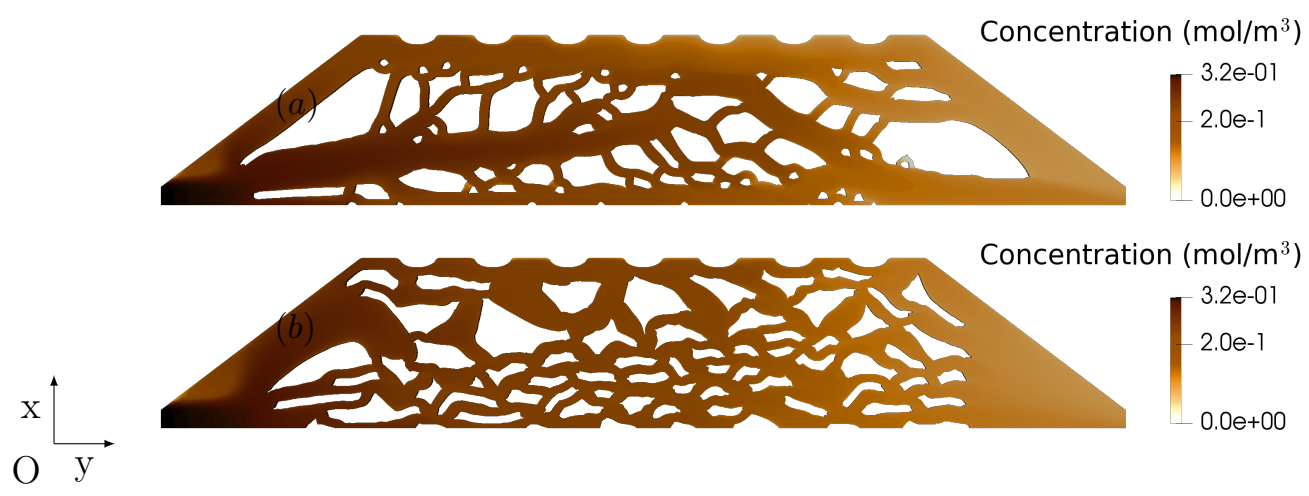

Figure 12: Optimized shapes with manufacturing constraints, (a) decreasing the maximum value of the energy contraint by a factor 2 and (b) increasing it by a factor 2 .

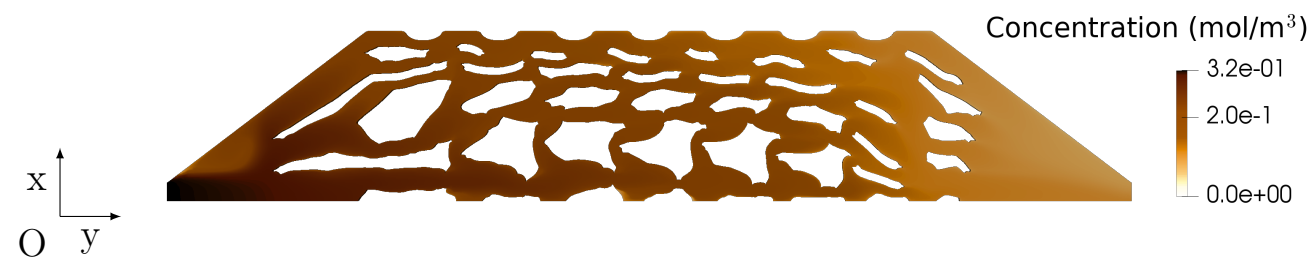

Figure 13: Optimized shapes modifying the topology of the initial reactor. 
bed reactor with a single-phase liquid flow involving a chemical reaction in laminar flow regime. Furthermore, the packing configuration which minimizes the average concentration of the reagent at the outlet of the reactor while satisfying the process model equations and the volume, the energy dissipation and the manufacturing constraints, was determined. However, the optimization algorithm developed exhibits some limitations which should be further investigated. Among these limitations, the treatment of the manufacturing constraints which is not robust. It suffers from too much rigidity since it does not allow constraints to be violated during the optimization process. Another limitation concerns the computation time which takes more than 2 days to converge (simulations performed on a $3.7 \mathrm{GHz}$ Xeon Dell Computer 5810). Parallel computing would not improve computational time because the method developed is an iterative method and the number of cells in the mesh is around 50000-70000. On the other hand, it is worth noticing that the use of a geometric algorithm does not allow us to modify the domain topology. Therefore, an interesting issue to be addressed is to allow topology changes within the optimization procedure.

As a close perspective, we are also currently working on generalizing our approach to other types of reactors, which requires to consider $3 \mathrm{D}$ geometries.

\section{A Proof of Theorem 2}

The well-posed character of System (16)-(17) is standard. Regarding (16), the proof is exactly similar to the one for System (3). We thus refer to [10, 19]. Regarding now System (17), we claim that the proof is a direct adaptation of [24, Prop. 1]. This point being clarified, it remains to express $J^{\prime}(\Omega)(\boldsymbol{\theta})$ under the form $\int_{\partial \Omega} G(\boldsymbol{\theta} \cdot \boldsymbol{n})$, where $G$ denotes the so-called shape gradient of $J$. We start from the expression (13) of $J^{\prime}(\Omega)(\boldsymbol{\theta})$.

Let us multiply the first equation of (17) by $\boldsymbol{U}^{\prime}$ and then integrate by parts. We get

$$
\begin{aligned}
-\nu \int_{\Omega} \Delta \boldsymbol{U}_{a} \cdot \boldsymbol{U}^{\prime} d x+\int_{\Omega}(\nabla \boldsymbol{U})^{\top} \boldsymbol{U}_{\boldsymbol{a}} \cdot \boldsymbol{U}^{\prime} d x-\int_{\Omega} \nabla \boldsymbol{U}_{\boldsymbol{a}} \boldsymbol{U} \cdot \boldsymbol{U}^{\prime} d x & \\
& \quad+\int_{\Omega} \nabla p_{a} \cdot \boldsymbol{U}^{\prime} d x=-\int_{\Omega} C_{a} \nabla C \cdot \boldsymbol{U}^{\prime} d x .
\end{aligned}
$$

Integrating each term by parts yields successively

$$
\begin{array}{rll}
-\nu \int_{\Omega} \Delta \boldsymbol{U}_{a} \cdot \boldsymbol{U}^{\prime} d x & \underset{(14 c)}{=} & 2 \nu \int_{\Omega} \boldsymbol{\varepsilon}\left(\boldsymbol{U}_{\boldsymbol{a}}\right): \boldsymbol{\varepsilon}\left(\boldsymbol{U}^{\prime}\right) d x-2 \nu \int_{\Gamma_{\text {out }} \cup \Gamma} \varepsilon\left(\boldsymbol{U}_{\boldsymbol{a}}\right) \boldsymbol{n} \cdot \boldsymbol{U}^{\prime} d \sigma \\
\int_{\Omega}(\nabla \boldsymbol{U})^{\top} \boldsymbol{U}_{\boldsymbol{a}} \cdot \boldsymbol{U}^{\prime} d x & \underset{(1 \mathrm{~d}) \underset{\text { and }}{=}(14 \mathrm{c})}{=} & -\int_{\Omega}\left(\nabla \boldsymbol{U}_{\boldsymbol{a}}\right) \boldsymbol{U}^{\prime} \cdot \boldsymbol{U} d x+\int_{\Gamma_{\text {out }}}\left(\boldsymbol{U} \cdot \boldsymbol{U}_{\boldsymbol{a}}\right)\left(\boldsymbol{U}^{\prime} \cdot \boldsymbol{n}\right) d \sigma \\
-\int_{\Omega}\left(\nabla \boldsymbol{U}_{\boldsymbol{a}}\right) \boldsymbol{U} \cdot \boldsymbol{U}^{\prime} d x & \underset{\text { (1d) and (14c) }}{=} & \int_{\Omega}\left(\nabla \boldsymbol{U}^{\prime}\right) \boldsymbol{U} \cdot \boldsymbol{U}_{\boldsymbol{a}} d x-\int_{\Gamma_{\text {out }}}(\boldsymbol{U} \cdot \boldsymbol{n})\left(\boldsymbol{U}^{\prime} \cdot \boldsymbol{U}_{\boldsymbol{a}}\right) d \sigma \\
\int_{\Omega} \nabla p_{a} \cdot \boldsymbol{U}^{\prime} d x & \underset{\text { (14b) and (14c) }}{=} & \int_{\Gamma_{\text {out }} \cup \Gamma} p_{a}\left(\boldsymbol{U}^{\prime} \cdot \boldsymbol{n}\right) d \sigma .
\end{array}
$$

It follows that

$$
\begin{aligned}
& \int_{\Omega}\left(2 \nu \varepsilon\left(\boldsymbol{U}_{\boldsymbol{a}}\right):\right.\left.\boldsymbol{\varepsilon}\left(\boldsymbol{U}^{\prime}\right)-\left(\nabla \boldsymbol{U}_{\boldsymbol{a}}\right) \boldsymbol{U}^{\prime} \cdot \boldsymbol{U}+\left(\nabla \boldsymbol{U}^{\prime}\right) \boldsymbol{U} \cdot \boldsymbol{U}_{\boldsymbol{a}}\right) d x \\
&-\int_{\Gamma_{\text {out }}}\left(\left(\boldsymbol{U} \cdot \boldsymbol{U}_{\boldsymbol{a}}\right)\left(\boldsymbol{U}^{\prime} \cdot \boldsymbol{n}\right)-(\boldsymbol{U} \cdot \boldsymbol{n})\left(\boldsymbol{U}^{\prime} \cdot \boldsymbol{U}_{\boldsymbol{a}}\right) \boldsymbol{\sigma}\left(\boldsymbol{U}_{\boldsymbol{a}}, p_{a}\right) \boldsymbol{n} \cdot \boldsymbol{U}^{\prime}\right) d \sigma \\
& \quad-\int_{\Gamma} \boldsymbol{\sigma}\left(\boldsymbol{U}_{\boldsymbol{a}}, p_{a}\right) \boldsymbol{n} \cdot \boldsymbol{U}^{\prime} d \sigma=-\int_{\Omega} C_{a} \nabla C \cdot \boldsymbol{U}^{\prime} d x .
\end{aligned}
$$


Let us multiply the first equation of (14) by $\boldsymbol{U}_{\boldsymbol{a}}$ and then integrate by parts on $\Omega$. One gets

$$
-\nu \int_{\Omega} \Delta \boldsymbol{U}^{\prime} \cdot \boldsymbol{U}_{\boldsymbol{a}} d x+\int_{\Omega}(\nabla \boldsymbol{U}) \boldsymbol{U}^{\prime} \cdot \boldsymbol{U}_{\boldsymbol{a}} d x+\int_{\Omega}\left(\nabla \boldsymbol{U}^{\prime}\right) \boldsymbol{U} \cdot \boldsymbol{U}_{\boldsymbol{a}} d x+\int_{\Omega} \nabla p^{\prime} \cdot \boldsymbol{U}_{\boldsymbol{a}} d x=0
$$

Integrating each term by parts yields successively

$$
\begin{array}{ccl}
-\nu \int_{\Omega} \Delta \boldsymbol{U}^{\prime} \cdot \boldsymbol{U}_{\boldsymbol{a}} d x & \underset{(17 c)}{=} & 2 \nu \int_{\Omega} \varepsilon\left(\boldsymbol{U}_{\boldsymbol{a}}\right): \boldsymbol{\varepsilon}\left(\boldsymbol{U}^{\prime}\right) d x-2 \nu \int_{\Gamma_{\text {out }}} \boldsymbol{\varepsilon}\left(\boldsymbol{U}^{\prime}\right) \boldsymbol{n} \cdot \boldsymbol{U}_{\boldsymbol{a}} d \sigma \\
\int_{\Omega}(\nabla \boldsymbol{U}) \boldsymbol{U}^{\prime} \cdot \boldsymbol{U}_{\boldsymbol{a}} d x & \begin{array}{c}
\text { (1d) and (14c) } \\
=
\end{array} & -\int_{\Omega}\left(\nabla \boldsymbol{U}_{\boldsymbol{a}}\right) \boldsymbol{U}^{\prime} \cdot \boldsymbol{U} d x+\int_{\Gamma_{\text {out }}}\left(\boldsymbol{U} \cdot \boldsymbol{U}^{\prime}\right)\left(\boldsymbol{U}_{\boldsymbol{a}} \cdot \boldsymbol{n}\right) d \sigma \\
\int_{\Omega} \nabla p^{\prime} \cdot \boldsymbol{U}_{\boldsymbol{a}} d x & \begin{array}{c}
=(17 b) \text { and (17c) } \\
=
\end{array} \int_{\Gamma_{\text {out }}} & p^{\prime}\left(\boldsymbol{U}_{\boldsymbol{a}} \cdot \boldsymbol{n}\right) d \sigma
\end{array}
$$

By taking into account the computations above and the boundary conditions (14e) on $\boldsymbol{U}^{\prime}$, we rewrite(26) as

$$
\begin{aligned}
\int_{\Omega}\left(2 \nu \boldsymbol{\varepsilon}\left(\boldsymbol{U}_{\boldsymbol{a}}\right): \boldsymbol{\varepsilon}\left(\boldsymbol{U}^{\prime}\right)-\left(\nabla \boldsymbol{U}_{\boldsymbol{a}}\right) \boldsymbol{U}^{\prime} \cdot \boldsymbol{U}+\left(\nabla \boldsymbol{U}^{\prime}\right) \boldsymbol{U} \cdot \boldsymbol{U}_{\boldsymbol{a}}\right) d x & \\
& +\int_{\Gamma_{\text {out }}}\left(\boldsymbol{U} \cdot \boldsymbol{U}_{\boldsymbol{a}}\right)\left(\boldsymbol{U}^{\prime} \cdot \boldsymbol{n}\right) d \sigma=0
\end{aligned}
$$

Combining (25) and (27) yields

$$
\int_{\Omega} C_{a} \nabla C \cdot \boldsymbol{U}^{\prime} d x=\int_{\Gamma \cup \Gamma_{\text {out }}} \boldsymbol{\sigma}\left(\boldsymbol{U}_{\boldsymbol{a}}, p_{a}\right) \boldsymbol{n} \cdot \boldsymbol{U}^{\prime} d \sigma-\int_{\Gamma_{\text {out }}}(\boldsymbol{U} \cdot \boldsymbol{n})\left(\boldsymbol{U}^{\prime} \cdot \boldsymbol{U}_{\boldsymbol{a}}\right) d \sigma
$$

Let us now use the same approach to deal with (15) and (16). We multiply the first equation of (16) by $C^{\prime}$ and then integrate by parts. We get

$$
-D \int_{\Omega} \Delta C_{a} C^{\prime} d x-\int_{\Omega}\left(\boldsymbol{U} \cdot \nabla C_{a}\right) C^{\prime} d x+k \int_{\Omega} C_{a} C^{\prime} d x=0
$$

Integrating each term by parts yields

$$
\begin{aligned}
-D \int_{\Omega} \Delta C_{a} C^{\prime} d x & =D \int_{\Omega}\left(\nabla C_{a} \cdot \nabla C^{\prime}\right) d x-D \int_{\partial \Omega} \frac{\partial C_{a}}{\partial n} C^{\prime} d \sigma \\
\int_{\Omega}\left(\boldsymbol{U} \cdot \nabla C_{a}\right) C^{\prime} d x & =-\int_{\Omega}\left(\boldsymbol{U} \cdot \nabla C^{\prime}\right) C_{a} d x+\int_{\partial \Omega}(\boldsymbol{U} \cdot \boldsymbol{n})\left(C_{a} C^{\prime}\right) d \sigma
\end{aligned}
$$

Thus, (29) rewrites

$$
\begin{aligned}
D \int_{\Omega}\left(\nabla C_{a} \cdot \nabla C^{\prime}\right) d x-D \int_{\partial \Omega} \frac{\partial C_{a}}{\partial n} C^{\prime} d \sigma+\int_{\Omega} & \left(\boldsymbol{U} \cdot \nabla C^{\prime}\right) C_{a} d x \\
& -\int_{\partial \Omega}(\boldsymbol{U} \cdot \boldsymbol{n})\left(C_{a} C^{\prime}\right) d \sigma+k \int_{\Omega} C_{a} C^{\prime} d x=0
\end{aligned}
$$

Similarly, let us multiply the first equation of (15) by $C_{a}$ and then integrate by parts. We obtain

$$
-D \int_{\Omega} \Delta C^{\prime} C_{a} d x+\int_{\Omega}\left(\boldsymbol{U} \cdot \nabla C^{\prime}\right) C_{a} d x+\int_{\Omega}\left(\boldsymbol{U}^{\prime} \cdot \nabla C\right) C_{a} d x+k \int_{\Omega} C_{a} C^{\prime} d x=0
$$


which also rewrites

$$
\begin{aligned}
D \int_{\Omega}\left(\nabla C_{a} \cdot \nabla C^{\prime}\right) d x-D \int_{\partial \Omega} \frac{\partial C^{\prime}}{\partial n} C_{a} d \sigma+\int_{\Omega}(\boldsymbol{U} & \left.\cdot \nabla C^{\prime}\right) C_{a} d x \\
& +\int_{\Omega}\left(\boldsymbol{U}^{\prime} \cdot \nabla C\right) C_{a} d x+k \int_{\Omega} C_{a} C^{\prime} d x=0
\end{aligned}
$$

Combining (30) and (32) leads to

$$
\int_{\Omega}\left(\boldsymbol{U}^{\prime} \cdot \nabla C\right) C_{a} d x+\underbrace{\int_{\partial \Omega}(\boldsymbol{U} \cdot \boldsymbol{n})\left(C_{a} C^{\prime}\right) d \sigma+D \int_{\partial \Omega} \frac{\partial C_{a}}{\partial n} C^{\prime} d \sigma-D \int_{\partial \Omega} \frac{\partial C^{\prime}}{\partial n} C_{a} d \sigma}_{\mathrm{I}}=0 .
$$

Observe that $\mathrm{I}$ has no contribution on $\Gamma_{\text {in }}$ and $\Gamma_{\text {lat }}$. Furthermore, one has

$$
\begin{aligned}
\mathrm{I}-\int_{\Gamma_{\text {out }}} C^{\prime} d \sigma & =-D \int_{\Gamma} C_{a}\left(-\frac{\partial C^{2}}{\partial n^{2}}(\boldsymbol{\theta} \cdot \boldsymbol{n})+\nabla C \cdot(\nabla(\boldsymbol{\theta} \cdot \boldsymbol{n})-(\nabla(\boldsymbol{\theta} \cdot \boldsymbol{n}) \cdot \boldsymbol{n}) \boldsymbol{n})\right) d \sigma \\
& =-D \int_{\Gamma} C_{a}\left(-\frac{\partial C^{2}}{\partial n^{2}}(\boldsymbol{\theta} \cdot \boldsymbol{n})+\nabla C \cdot \nabla(\boldsymbol{\theta} \cdot \boldsymbol{n})-\nabla C \cdot(\nabla(\boldsymbol{\theta} \cdot \boldsymbol{n}) \cdot \boldsymbol{n}) \boldsymbol{n}\right) d \sigma \\
& =-D \int_{\Gamma} C_{a}\left(-\frac{\partial C^{2}}{\partial n^{2}}(\boldsymbol{\theta} \cdot \boldsymbol{n})+\nabla C \cdot \nabla(\boldsymbol{\theta} \cdot \boldsymbol{n})-(\nabla(\boldsymbol{\theta} \cdot \boldsymbol{n}) \cdot \boldsymbol{n})(\nabla C \cdot \boldsymbol{n})\right) d \sigma \\
& =-D \int_{\Gamma} C_{a}\left(-\frac{\partial C^{2}}{\partial n^{2}}(\boldsymbol{\theta} \cdot \boldsymbol{n})+\nabla C \cdot \nabla(\boldsymbol{\theta} \cdot \boldsymbol{n})-\frac{\partial(\boldsymbol{\theta} \cdot \boldsymbol{n})}{\partial n} \frac{\partial C}{\partial n}\right) d \sigma
\end{aligned}
$$

according to (15). Since $\Gamma$ is the only part of the boundary of $\Omega$ met by the support of $\boldsymbol{\theta}$, it is legitimate to use an integration by parts formula ${ }^{3}$ on $\partial \Omega$. It yields

$$
\mathrm{I}-\int_{\Gamma_{\text {out }}} C^{\prime} d \sigma=D \int_{\Gamma} C_{a}\left(\Delta C(\boldsymbol{\theta} \cdot \boldsymbol{n})-\mathcal{H} \frac{\partial C}{\partial n}(\boldsymbol{\theta} \cdot \boldsymbol{n})\right) \underset{(3 \mathrm{c})}{=} D \int_{\Gamma} C_{a} \Delta C(\boldsymbol{\theta} \cdot \boldsymbol{n})
$$

Hence, (33) reads

$$
\int_{\Omega}\left(\boldsymbol{U}^{\prime} \cdot \nabla C\right) C_{a} d x+J^{\prime}(\Omega)(\boldsymbol{\theta})+\mathcal{D} \int_{\Gamma} C_{a} \Delta C(\boldsymbol{\theta} \cdot \boldsymbol{n})=0
$$

By combining (28) and (36), we obtain

$$
\begin{aligned}
J^{\prime}(\Omega)(\boldsymbol{\theta})= & -\int_{\Gamma \cup \Gamma_{\text {out }}} \boldsymbol{\sigma}\left(\boldsymbol{U}_{\boldsymbol{a}}, p_{a}\right) \cdot n \cdot \boldsymbol{U}^{\prime} d \sigma-D \int_{\Gamma} C_{a} \Delta C(\boldsymbol{\theta} \cdot \boldsymbol{n}) \\
& -\int_{\Gamma_{\text {out }}}(\boldsymbol{U} \cdot \boldsymbol{n})\left(\boldsymbol{U}^{\prime} \cdot \boldsymbol{U}_{\boldsymbol{a}}\right) d \sigma .
\end{aligned}
$$

${ }^{3}$ Recall that if $f \in H^{2}(\Omega)$ and $g \in H^{3}(\Omega)$, then one has

$$
\int_{\partial \Omega} \nabla f \cdot \nabla g d \sigma=-\int_{\partial \Omega} f \Delta g d \sigma+\int_{\partial \Omega}\left(\frac{\partial f}{\partial n} \frac{\partial g}{\partial n}+f \frac{\partial^{2} g}{\partial n^{2}}+\mathcal{H} f \frac{\partial g}{\partial n}\right) d \sigma
$$

where $\mathcal{H}$ denotes the mean curvature on $\partial \Omega$ (see [23, Chapter 5]). 
Finally, using the boundary conditions (14c), (14d) and (17d) leads to

$$
J^{\prime}(\Omega)(\boldsymbol{\theta})=\int_{\Gamma}\left(\left(2 \nu \boldsymbol{\varepsilon}\left(\boldsymbol{U}_{\boldsymbol{a}}\right) \boldsymbol{n}-p_{a} \boldsymbol{n}\right) \cdot \frac{\partial \boldsymbol{U}}{\partial n}-D C_{a} \Delta C\right)(\boldsymbol{\theta} \cdot \boldsymbol{n}) d \sigma
$$

To conclude, it remains to rewrite the expression above in a more symmetrical way. To this aim, we will use the following lemma whose proof is postponed at the en $\mathrm{d}$ of this section for the sake of clarity.

Lemma 1. Assume that $\partial \Omega$ is smooth enough so that $\boldsymbol{U}$ and $\boldsymbol{U}_{\boldsymbol{a}}$ belong to $H^{2}(\Omega)$. Thus, one has on $\Gamma$

$$
\begin{gathered}
\boldsymbol{n} \cdot \frac{\partial \boldsymbol{U}}{\partial n}=0 \\
\boldsymbol{\varepsilon}(\boldsymbol{U}) \boldsymbol{n} \cdot \frac{\partial \boldsymbol{U}}{\partial n}=|\varepsilon(\boldsymbol{U})|^{2} \\
\left(\varepsilon\left(\boldsymbol{U}_{\boldsymbol{a}}\right) \boldsymbol{n}\right) \cdot \frac{\partial \boldsymbol{U}}{\partial n}=\boldsymbol{\varepsilon}(\boldsymbol{U}): \boldsymbol{\varepsilon}\left(\boldsymbol{U}_{\boldsymbol{a}}\right)
\end{gathered}
$$

Roughly speaking, this follows from the fact that $\left(\boldsymbol{U}\right.$ and $\left.\boldsymbol{U}_{\boldsymbol{a}}\right)$ are divergence-free in $\Omega$ and vanish on $\Gamma$. Finally, this leads to the desired expression of the shape derivative:

$$
J^{\prime}(\Omega)(\boldsymbol{\theta})=\int_{\Omega}\left(2 \nu\left(\varepsilon(\boldsymbol{U}): \boldsymbol{\varepsilon}\left(\boldsymbol{U}_{\boldsymbol{a}}\right)-D C_{a} \Delta C\right)(\boldsymbol{\theta} \cdot \boldsymbol{n}) d \sigma\right.
$$

Proof of Lemma 1. Let us first show (39). Since $\boldsymbol{U}$ vanishes on $\Gamma$, one has $\nabla \boldsymbol{U}=(\nabla \boldsymbol{U n}) \boldsymbol{n}$. It follows that $\partial U_{i} / \partial x_{i}=n_{i} \partial U_{i} / \partial n$ and therefore

$$
\boldsymbol{n} \cdot \frac{\partial \boldsymbol{U}}{\partial n}=\sum_{i} n_{i} \frac{\partial U_{i}}{\partial n}=\sum_{i} \frac{\partial U_{i}}{\partial x_{i}}=\operatorname{div}(\boldsymbol{U})=0 \quad \text { on } \Gamma .
$$

Let us now prove (41). Using the same tricks as above enable us to write

$$
\frac{\partial U_{i}}{\partial x_{j}}=\frac{\partial U_{i}}{\partial n} n_{j} \quad \text { and } \quad \operatorname{div} \boldsymbol{U}=\sum_{i} \frac{\partial U_{i}}{\partial x_{i}}=\sum_{i} \frac{\partial U_{i}}{\partial n} n_{i}=0
$$

from which we infer that

$$
\begin{aligned}
\varepsilon(\boldsymbol{U}) \boldsymbol{n} & =\frac{1}{2}\left(\sum_{j}\left(\frac{\partial U_{i}}{\partial x_{j}}+\frac{\partial U_{j}}{\partial x_{i}}\right) n_{j}\right)_{i}=\frac{1}{2}\left(\sum_{j} \frac{\partial U_{i}}{\partial n} n_{j}^{2}+\frac{\partial U_{j}}{\partial n} n_{i} n_{j}\right)_{i} \\
& =\frac{1}{2}\left(\frac{\partial U_{i}}{\partial n} \sum_{j} n_{j}^{2}\right)_{i}=\frac{1}{2}\left(\frac{\partial U_{i}}{\partial n}\right)_{i}=\frac{1}{2} \frac{\partial \boldsymbol{U}}{\partial \boldsymbol{n}}
\end{aligned}
$$

on $\Gamma$. It follows that $\left(\varepsilon\left(\boldsymbol{U}_{\boldsymbol{a}}\right) \boldsymbol{n}\right) \cdot \frac{\partial \boldsymbol{U}}{\partial n}=2\left(\varepsilon\left(\boldsymbol{U}_{\boldsymbol{a}}\right) \boldsymbol{n}\right) \cdot(\varepsilon(\boldsymbol{U}) \boldsymbol{n})$ on $\Gamma$ and then

$$
\begin{aligned}
\left(\varepsilon\left(\boldsymbol{U}_{\boldsymbol{a}}\right) \boldsymbol{n}\right) \cdot \frac{\partial \boldsymbol{U}}{\partial n} & =\frac{1}{2} \sum_{i} \sum_{j}\left(\frac{\partial U_{a_{i}}}{\partial x_{j}}+\frac{\partial U_{a_{j}}}{\partial x_{i}}\right) n_{j} \sum_{l}\left(\frac{\partial U_{i}}{\partial x_{l}}+\frac{\partial U_{l}}{\partial x_{i}}\right) n_{l} \\
& =\frac{1}{2} \sum_{i, j, l}\left(\frac{\partial U_{a_{i}}}{\partial \boldsymbol{n}} n_{j}^{2}+\frac{\partial U_{a_{j}}}{\partial \boldsymbol{n}} n_{i} n_{j}\right)\left(\frac{\partial U_{i}}{\partial \boldsymbol{n}} n_{l}^{2}+\frac{\partial U_{l}}{\partial \boldsymbol{n}} n_{i} n_{l}\right) \\
& =\frac{1}{2} \sum_{i, j, l}(\frac{\partial U_{a_{i}}}{\partial \boldsymbol{n}} \frac{\partial U_{i}}{\partial \boldsymbol{n}} n_{j}^{2} n_{l}^{2}+\underbrace{\frac{\partial U_{a_{j}}}{\partial \boldsymbol{n}} n_{j}}_{\operatorname{div} \boldsymbol{U}_{\boldsymbol{a}}=0} \underbrace{\frac{\partial U_{i}}{\partial \boldsymbol{n}} n_{i}}_{\operatorname{div} \boldsymbol{U}=0} n_{l}^{2}+\frac{\partial U_{a_{i}}}{\partial \boldsymbol{n}} n_{i} \frac{\partial U_{l}}{\partial \boldsymbol{n}} n_{l} n_{j}^{2}+\frac{\partial U_{a_{j}}}{\partial \boldsymbol{n}} n_{j} \frac{\partial U_{l}}{\partial \boldsymbol{n}} n_{l} n_{i}^{2}) .
\end{aligned}
$$


The three last terms of the sum vanish ( $\operatorname{since} \operatorname{div} \boldsymbol{U}=\operatorname{div} \boldsymbol{U}_{\boldsymbol{a}}=0$ on $\Gamma$ ) and then,

$$
2\left(\varepsilon\left(\boldsymbol{U}_{\boldsymbol{a}}\right) \boldsymbol{n}\right) \cdot(\varepsilon(\boldsymbol{U}) \boldsymbol{n})=\frac{1}{2} \sum_{i} \frac{\partial U_{a_{i}}}{\partial \boldsymbol{n}} \frac{\partial U_{i}}{\partial \boldsymbol{n}}
$$

On the other hand, one has

$$
\begin{aligned}
\boldsymbol{\varepsilon}(\boldsymbol{U}): \boldsymbol{\varepsilon}\left(\boldsymbol{U}_{\boldsymbol{a}}\right) & =\frac{1}{4} \sum_{i, j}\left(\frac{\partial U_{i}}{\partial x_{j}}+\frac{\partial U_{j}}{\partial x_{i}}\right)\left(\frac{\partial U_{a_{i}}}{\partial x_{j}}+\frac{\partial U_{a_{j}}}{\partial x_{i}}\right) \\
& =\frac{1}{4} \sum_{i, j}\left(\frac{\partial U_{i}}{\partial x_{j}} \frac{\partial U_{a_{i}}}{\partial x_{j}}+\frac{\partial U_{j}}{\partial x_{i}} \frac{\partial U_{a_{i}}}{\partial x_{j}}+\frac{\partial U_{i}}{\partial x_{j}} \frac{\partial U_{a_{j}}}{\partial x_{i}}+\frac{\partial U_{j}}{\partial x_{i}} \frac{\partial U_{a_{j}}}{\partial x_{i}}\right) \\
& =\frac{1}{2} \sum_{i, j}\left(\frac{\partial U_{i}}{\partial x_{j}} \frac{\partial U_{a_{i}}}{\partial x_{j}}+\frac{\partial U_{j}}{\partial x_{i}} \frac{\partial U_{a_{i}}}{\partial x_{j}}\right)=\frac{1}{2} \sum_{i, j}\left(\frac{\partial U_{i}}{\partial \boldsymbol{n}} \frac{\partial U_{a_{i}}}{\partial \boldsymbol{n}} n_{j}^{2}+\frac{\partial U_{j}}{\partial \boldsymbol{n}} \frac{\partial U_{a_{i}}}{\partial \boldsymbol{n}} n_{i} n_{j}\right) \\
& =\frac{1}{2} \sum_{i} \frac{\partial U_{i}}{\partial \boldsymbol{n}} \frac{\partial U_{a_{i}}}{\partial \boldsymbol{n}} \underbrace{\sum_{j} n_{j}^{2}}_{=1}+\frac{1}{2} \underbrace{\sum_{j} \frac{\partial U_{j}}{\partial \boldsymbol{n}} n_{j}}_{\operatorname{div} \boldsymbol{U}=0}+\frac{1}{2} \underbrace{\sum_{i} \frac{\partial U_{a_{i}}}{\partial \boldsymbol{n}} n_{i}}_{\operatorname{div} \boldsymbol{U}_{\boldsymbol{a}}=0}=\frac{1}{2} \sum_{i} \frac{\partial U_{i}}{\partial \boldsymbol{n}} \frac{\partial U_{a_{i}}}{\partial \boldsymbol{n}},
\end{aligned}
$$

whence (41). The proof of (40) is exactly similar.

\section{B Proof of Proposition 1}

Let us first recall (see for instance $[1,23,14]$ ) that

$$
\begin{aligned}
C_{1}^{\prime}(\Omega)(\boldsymbol{\theta}) & =\frac{d}{d t}\left(\left.\int_{\Omega_{t}} 1 d x\right|_{t=0}-\nu\left(\Omega_{0}\right)\right)=\int_{\partial \Omega}(\boldsymbol{\theta} \cdot \boldsymbol{n}) d \sigma \\
C_{2}^{\prime}(\Omega)(\boldsymbol{\theta}) & =\frac{d}{d t}\left(\left.2 \nu \int_{\Omega_{t}}|\varepsilon(\boldsymbol{U})|^{2} d x\right|_{t=0}-\mathcal{E}\left(\Omega_{0}\right)\right) \\
& =2 \nu \int_{\partial \Omega}|\varepsilon(\boldsymbol{U})|^{2}(\boldsymbol{\theta} \cdot \boldsymbol{n}) d \sigma+4 \nu \int_{\Omega} \boldsymbol{\varepsilon}(\boldsymbol{U}): \boldsymbol{\varepsilon}\left(\boldsymbol{U}^{\prime}\right) d x
\end{aligned}
$$

It is standard to rewrite $C_{2}^{\prime}(\Omega)(\boldsymbol{\theta})$ in a more workable form, by introducing a well-chosen adjoint method. In accordance with [14], let us introduce the adjoint state $\left(\boldsymbol{U}_{\boldsymbol{a}}, p_{a}\right)$ solving the linearized Navier-Stokes system

$$
\begin{aligned}
-\nu \Delta \boldsymbol{U}_{\boldsymbol{a}}+(\nabla \boldsymbol{U})^{\top} \boldsymbol{U}_{\boldsymbol{a}}-\nabla \boldsymbol{U}_{\boldsymbol{a}} \boldsymbol{U}+\nabla p_{a}=-\lambda_{\varepsilon} 2 \nu \Delta \boldsymbol{U} & \text { in } \Omega \\
\nabla \cdot \boldsymbol{U}_{\boldsymbol{a}}=0 & \text { in } \Omega \\
\boldsymbol{U}_{\boldsymbol{a}}=0 & \text { on } \Gamma_{\text {in }} \cup \Gamma_{\text {lat }} \cup \Gamma \\
\boldsymbol{\sigma}\left(\boldsymbol{U}_{\boldsymbol{a}}, p_{a}\right) \boldsymbol{n}+(\boldsymbol{U} \cdot \boldsymbol{n}) \boldsymbol{U}_{\boldsymbol{a}}=4 \nu \lambda_{\varepsilon} \boldsymbol{\varepsilon}(\boldsymbol{U}) \boldsymbol{n} & \text { on } \Gamma_{\text {out }}
\end{aligned}
$$

Hence, one shows with the help of well-adapted integrations by $\operatorname{parts}^{4}$ that the quantity

${ }^{4}$ More precisely, we use the adapted formula

$$
\forall(\mathbf{u}, \mathbf{v}) \in\left[H^{2}(\Omega)\right]^{2}, \int_{\Omega} \varepsilon(\mathbf{u}): \varepsilon(\mathbf{v}) \mathrm{d} x=-\int_{\Omega}(\Delta \mathbf{u}+\nabla(\nabla \cdot \mathbf{u})) \cdot \mathbf{v} \mathrm{d} x+\int_{\partial \Omega} \varepsilon(\mathbf{u}) \cdot \mathbf{n} \cdot \mathbf{v} \mathrm{d} s,
$$

where $\Omega$ is a bounded regular domain of $\mathbb{R}^{2}$. We refer fo instance to $[11,8]$. 
$C_{2}^{\prime}(\Omega)(\boldsymbol{\theta})$ rewrites

$$
C_{2}^{\prime}(\Omega)(\boldsymbol{\theta})=\int_{\Omega}\left(2 \nu\left(\varepsilon(\boldsymbol{U}): \varepsilon\left(\boldsymbol{U}_{\boldsymbol{a}}\right)-\lambda_{\varepsilon} \boldsymbol{\varepsilon}(\boldsymbol{U}): \boldsymbol{\varepsilon}(\boldsymbol{U})\right)\right)(\boldsymbol{\theta} \cdot \boldsymbol{n}) d \sigma
$$

The expected conclusion hence follows by combining these formula with the result of Proposition 2 .

\section{References}

[1] G. Allaire, Conception optimale de structures, vol. 58 of Collection Mathématiques et Applications, Springer, 2007.

[2] G. Allaire, C. Dapogny, R. Estevez, A. Faure, and G. Michailidis, Structural optimization under overhang constraints imposed by additive manufacturing processes: an overview of some recent results, Appl. Math. Nonlinear Sci., 2 (2017), pp. 385402, https://doi.org/10.21042/AMNS.2017.2.00031, https://doi.org/10.21042/AMNS. 2017.2 .00031$.

[3] G. Allaire, C. Dapogny, R. Estevez, A. Faure, and G. Michailidis, Structural optimization under overhang constraints imposed by additive manufacturing technologies, J. Comput. Phys., 351 (2017), pp. 295-328, https://doi.org/10.1016/j.jcp.2017.09.041, https://doi.org/10.1016/j.jcp.2017.09.041.

[4] G. Allaire, C. Dapogny, A. Faure, and G. Michailidis, Shape optimization of a layer by layer mechanical constraint for additive manufacturing, C. R. Math. Acad. Sci. Paris, 355 (2017), pp. 699-717, https://doi.org/10.1016/j.crma.2017.04.008, https://doi.org/ $10.1016 / j$.crma.2017.04.008.

[5] G. Allaire, F. Jouve, and G. Michailidis, Thickness control in structural optimization via a level set method, Struct. Multidiscip. Optim., 53 (2016), pp. 1349-1382, https://doi. org/10.1007/s00158-016-1453-y, https://doi.org/10.1007/s00158-016-1453-y.

[6] M. A. Ansari AND K.-Y. Kim, Shape optimization of a micromixer with staggered herringbone groove, Chemical Engineering Science, 62 (2007), pp. 6687-6695, https://doi. org $/ 10.1016 / j$.ces .2007 .07 .059 .

[7] D. Attali, Squelettes et graphes de Voronoi 2D et 3D, PhD thesis, 1995.

[8] M. Bergounioux and Y. Privat, Shape optimization with Stokes constraints over the set of axisymmetric domains, SIAM J. Control Optim., 51 (2013), pp. 599-628, https://doi. org/10.1137/100818133, https://doi.org/10.1137/100818133.

[9] M. Bonnivard, F. Omnès, And Y. Privat, Modeling and optimization of hourglass-shaped aquaporins, Math. Models Methods Appl. Sci., 28 (2018), pp. 1529-1564, https://doi.org/ 10.1142/S0218202518500422, https://doi.org/10.1142/S0218202518500422.

[10] F. Boyer, Trace theorems and spatial continuity properties for the solutions of the transport equation, Differential Integral Equations, 18 (2005), pp. 891-934.

[11] F. Boyer and P. Fabrie, Mathematical tools for the study of the incompressible Navier-Stokes equations and related models, vol. 183 of Applied Mathematical Sciences, Springer, New York, 2013, https://doi.org/10.1007/978-1-4614-5975-0, https://doi. org/10.1007/978-1-4614-5975-0. 
[12] G. W. Burgreen, O. Baysal, and M. E. Eleshaky, Improving the efficiency of aerodynamic shape optimization, AIAA journal, 32 (1994), pp. 69-76, https://doi.org/ $10.2514 / 3.11952$.

[13] H. W. Carlson and W. D. Middleton, A numerical method for the design of camber surfaces of supersonic wings with arbitrary planforms, National Aeronautics and Space Administration, 1964.

[14] C. Dapogny, P. Frey, F. Omnès, and Y. Privat, Geometrical shape optimization in fluid mechanics using FreeFem++, Struct. Multidiscip. Optim., 58 (2018), pp. 2761-2788, https://doi.org/10.1007/s00158-018-2023-2, https://doi.org/10. $1007 /$ s00158-018-2023-2.

[15] X. D. De La Sablonière, B. Mauroy, and Y. Privat, Shape minimization of the dissipated energy in dyadic trees, Discrete Contin. Dyn. Syst. Ser. B, 16 (2011), pp. 767-799, https://doi.org/10.3934/dcdsb.2011.16.767, https://doi.org/10.3934/dcdsb.2011. 16.767 .

[16] G. Dogan, P. Morin, R. H. Nochetto, and M. Verani, Discrete gradient flows for shape optimization and applications, Computer Methods in Applied Mechanics and Engineering, 196 (2007), pp. 3898-3914.

[17] J. Droniou, Solving convection-diffusion equations with mixed, Neumann and Fourier boundary conditions and measures as data, by a duality method, Adv. Differential Equations, 5 (2000), pp. 1341-1396.

[18] H. Federer, Curvature measures, Trans. Amer. Math. Soc., 93 (1959), pp. 418-491, https: //doi.org/10.2307/1993504, https://doi.org/10.2307/1993504.

[19] F. Feppon, G. Allaire, F. Bordeu, J. Cortial, and C. Dapogny, Shape optimization of a coupled thermal fluid-structure problem in a level set mesh evolution framework, SeMA J., 76 (2019), pp. 413-458, https://doi.org/10.1007/s40324-018-00185-4, https://doi. org/10.1007/s40324-018-00185-4.

[20] A. V. Fursikov and R. Rannacher, Optimal Neumann Control for the Two-dimensional Steady-state Navier-Stokes equations, Birkhäuser Basel, Basel, 2010, pp. 193-221, https://doi.org/10.1007/978-3-0346-0152-8_11, https://doi.org/10.1007/ 978-3-0346-0152-8_11.

[21] G. P. GALDI, An introduction to the mathematical theory of the Navier-Stokes equations, Springer Monographs in Mathematics, Springer, New York, second ed., 2011, https:// doi.org/10.1007/978-0-387-09620-9, https://doi.org/10.1007/978-0-387-09620-9. Steady-state problems.

[22] I. P. R. Grundtvig, A. E. Dauganrd, J. M. Woodley, K. V. Gernaey, and U. KRÜHNE, Shape optimization as a tool to design biocatalytic microreactors, Chemical Engineering Journal, 322 (2017), pp. 215-223, https ://doi.org/10.1016/j.cej.2017.03.045.

[23] A. Henrot and M. Pierre, Shape variation and optimization: a geometrical analysis, vol. 28 of Tracts in Mathematics, European Mathematical Society, Zurich, 2018.

[24] A. Henrot and Y. Privat, What is the optimal shape of a pipe?, Arch. Ration. Mech. Anal., 196 (2010), pp. 281-302, https://doi.org/10.1007/s00205-009-0243-8, https: //doi.org/10.1007/s00205-009-0243-8. 
[25] R. M. Hicks And P. A. Henne, Wing design by numerical optimization, Journal of Aircraft, 15 (1978), pp. 407-412.

[26] G. Holzinger, Openfoam: A little user-manual, CD-Laboratory-Particulate Flow Modelling, Johannes Keplper University: Linz, Austria, (2015).

[27] C. LE Roux AND B. RedDy, The steady navier-stokes equations with mixed boundary conditions: application to free boundary flows, Nonlinear Analysis: Theory, Methods \& Applications, 20 (1993), pp. 1043 - 1068, https://doi.org/https: //doi.org/10.1016/0362-546X(93)90094-9, http://www.sciencedirect.com/science/ article/pii/0362546X93900949.

[28] R. Liang And Z. Yuan, Computational shape optimization of microreactors based on cfd simulation and surrogate model driven optimization, in Computer Aided Chemical Engineering, vol. 48, Elsevier, 2020, pp. 925-930, https://doi.org/10.1016/B978-0-12-823377-1. 50155-5.

[29] B. Mohammadi and O. Pironneau, Applied shape optimization for fluids, Oxford University Press, 2010.

[30] F. Moukalled, L. Mangani, M. Darwish, et al., The finite volume method in computational fluid dynamics, vol. 6, Springer, 2016.

[31] F. Murat and J. Simon, Sur le contrôle par un domaine géométrique, Pré-publication du Laboratoire d'Analyse Numérique,(76015), (1976).

[32] J. Reuther, J. J. Alonso, M. J. Rimlinger, and A. Jameson, Aerodynamic shape optimization of supersonic aircraft configurations via an adjoint formulation on distributed memory parallel computers, Computers \& fluids, 28 (1999), pp. 675-700, https://doi.org/ 10.2514/6.1996-4045.

[33] G. SAVARÉ, Regularity results for elliptic equations in lipschitz domains, Journal of Functional Analysis, 152 (1998), pp. 176 - 201, https://doi.org/https://doi.org/10.1006/jfan. 1997.3158, http://www.sciencedirect.com/science/article/pii/S002212369793158X.

[34] J. Sokolowski And J.-P. ZolÉsio, Introduction to shape optimization: shape sensitivity analysis, Springer, 1992.

[35] R. Temam, Navier-Stokes equations, vol. 2 of Studies in Mathematics and its Applications, North-Holland Publishing Co., Amsterdam, third ed., 1984. Theory and numerical analysis, With an appendix by F. Thomasset.

[36] O. Tonomura, M. Kano, and S. Hasebe, Shape optimization of microchannels using cfd and adjoint method, in 20th European Symposium on Computer Aided Process Engineering, S. Pierucci and G. B. Ferraris, eds., vol. 28 of Computer Aided Chemical Engineering, Elsevier, 2010, pp. 37 - 42, https://doi.org/https://doi.org/10.1016/S1570-7946(10) 28007-0, http://www.sciencedirect.com/science/article/pii/S1570794610280070.

[37] H. G. Weller, G. Tabor, H. Jasak, and C. Fureby, A tensorial approach to computational continuum mechanics using object-oriented techniques, Computers in physics, 12 (1998), pp. 620-631. 